\title{
THE PROGRAM ITERATIONS METHOD IN GAME PROBLEM OF GUIDANCE AND SET-VALUED QUASISTRATEGIES ${ }^{1}$
}

\author{
Alexander G. Chentsov \\ Krasovskii Institute of Mathematics and Mechanics, \\ Ural Branch of Russian Academy of Sciences, Ekaterinburg, Russia, \\ chentsov@imm.uran.ru
}

\begin{abstract}
We consider a differential game of guidance-evasion, which is solved with the program iterations method. The iterated procedure is realized in the space of sets, the elements of which are the game's positions. The objective of this procedure is to construct the alternative partition of the space of positions as established by N.N. Krasovskii and A.I. Subbotin. In addition, more general assumptions on topological properties of the set defining the phase constraints are considered. The connection with the game's solution in the class of quasistrategies is investigated. These quasistrategies are defined as set-valued mappings on spaces of strategic (countably additive) Borel measures.
\end{abstract}

Key words: Differential game, Iterated procedure, Quasistrategy.

\section{Introduction}

Researche in the theory of differential games is motivated by many applied problems in this connection, see the known monograph [1], in which many practical problems with game elements are considered. However, the construction of the corresponding theory faces serious difficulties of mathematical character. We note that N.N. Krasovskii and his scientific school have done much to overcome many of those difficulties. In particular, in [2], a rigorous theory of differential games was constructed. A very important fragment of this theory is connected with the alternative theorem of N.N. Krasovskii and A.I. Subbotin (see $[2,3]$ ). This theorem defined the consequent development of the whole theory of differential games. In particular, the existence of a saddle point in the class of positional strategies was established (see [2]).

In connection with the alternative theorem, a very important role belongs to the methods that construct the set of positional absorption (see [2]) in the sense of solvability of the approach problem (more exactly, the problem of approaching the target set). One of such methods is realized by program iterations (see [4-8] and other). In this study, we consider a variant of the program iterations method connected with a construction of the set of positional absorption; in this connection, we note article [5], where an iterated procedure was realized in the space of sets (elements of which are positions of the game). However, in the present paper, this procedure is investigated under more general conditions (in this connection, see also [9]). In particular, we consider a basic game problem under the condition of generalized uniqueness; the latter condition was used by A.V. Kryazhimskii [10] under an important generalization of the alternative theorem.

\section{General notation}

We use the standard set-theoretical notation (quantifiers and propositional connectives; by $\emptyset$ we denote the empty set). In the following, $\exists$ ! replaces the expression "there exists a unique", and $\triangleq$ means equality by definition. The set, whose elements are themselves sets, we call further the family. We follow here the axiom of choice. For an object $z$, by $\{z\}$ we denote the singleton containing $z$.

\footnotetext{
${ }^{1}$ This work was supported by Russian Foundation for Basic Research (projects no. 16-01-00649 and no. 16-01-00505)
} 
If $H$ is a set, by $\mathcal{P}(H)$ we denote the family of all subsets of $H ; \mathcal{P}^{\prime}(H) \triangleq \mathcal{P}(H) \backslash\{\emptyset\}$ (the family of all nonempty subsets of $H)$. Moreover, by $\operatorname{Fin}(H)$ we denote the family of all finite sets from $\mathcal{P}^{\prime}(H)$; here, $H$ is an arbitrary set. If $\mathcal{X}$ is a nonempty family and $Y$ is a set, then

$$
\left.\mathcal{X}\right|_{Y} \triangleq\{X \cap Y: X \in \mathcal{X}\} \in \mathcal{P}^{\prime}(\mathcal{P}(Y))
$$

in the following, (1.1) is used for the case when $\mathcal{X}$ is a topology of some set $\mathbb{X}$ and $Y \in \mathcal{P}(\mathbb{X})$. In the latter case, we obtain a topology of $Y$ induced by the topological space (TS) $(\mathbb{X}, \mathcal{X})$; in addition, $\left(Y,\left.\mathcal{X}\right|_{Y}\right)$ is a subspace of $(\mathbb{X}, \mathcal{X})$. We denote also as $\left.\mathcal{X}\right|_{Y}$ a relative topology of $Y$. If $(E, \tau)$ is a TS and $A \in \mathcal{P}(E)$, then, by $\operatorname{cl}(A, \tau)$, denote the closure of $A$ in the TS $(E, \tau)$. Moreover, for every TS $(E, \tau)$, by $(\tau-\operatorname{comp})[E]$, we denote the family of all nonempty compact (in $(E, \tau))$ subsets of $E$.

For all nonempty sets $A$ and $B$, by $B^{A}$ we denote the set of all mappings from $A$ into $B$; as usual, for $\mathbf{f} \in B^{A}$ and $a \in A$, by $\mathbf{f}(a), \mathbf{f}(a) \in B$, we denote the value of the mapping $\mathbf{f}$ at the point $a$. For some nonempty sets $A$ and $B$, a mapping $\mathbf{f} \in B^{A}$, and a set $C \in \mathcal{P}^{\prime}(A)$, we denote by $(\mathbf{f} \mid C)$ the restriction of $\mathbf{f}$ onto $C$; namely,

$$
(\mathbf{f} \mid C) \in B^{C}:(\mathbf{f} \mid C)(x) \triangleq \mathbf{f}(x) \quad \forall x \in C .
$$

If $E$ is a set and $\mathcal{E} \in \mathcal{P}(\mathcal{P}(E))$, then we assume that $\sigma_{E}^{o}(\mathcal{E})$ is a $\sigma$-algebra (or $\sigma$-field) of subsets of $E$ generated by $\mathcal{E}$. Namely, $\sigma_{E}^{o}(\mathcal{E})$ is the least $\sigma$-algebra of subsets of $E$ that still satisfies the relation $\mathcal{E} \subset \sigma_{E}^{o}(\mathcal{E})$.

We note some simple properties connected with passing to subspaces of a measurable space. Namely, for every set $X, \mathcal{X} \in \mathcal{P}^{\prime}(\mathcal{P}(X))$, and $Y \in \mathcal{P}(X)$, the equality

$$
\sigma_{Y}^{o}\left(\left.\mathcal{X}\right|_{Y}\right)=\left.\sigma_{X}^{o}(\mathcal{X})\right|_{Y}
$$

holds; if $Y \in \sigma_{X}^{o}(\mathcal{X})$, then we obtain the following equality:

$$
\sigma_{Y}^{o}\left(\left.\mathcal{X}\right|_{Y}\right)=\left\{\Sigma \in \sigma_{X}^{o}(\mathcal{X}) \mid \Sigma \subset Y\right\}
$$

in connection with (1.2) and (1.3), see [11, §2.3]. Obviously, a topology of $X$ can be used as a family $\mathcal{X}$.

In the following, $\mathbb{R}$ is the real line, $\mathbb{N} \triangleq\{1 ; 2 ; \ldots\} \in \mathcal{P}^{\prime}(\mathbb{R})$, and $\mathbb{N}_{o} \triangleq\{0\} \cup \mathbb{N}=\{0 ; 1 ; 2 ; \ldots\}$. For $k \in \mathbb{N}_{o}$ and $l \in \mathbb{N}_{o}$, we assume that

$$
\overline{k, l} \triangleq\left\{j \in \mathbb{N}_{o} \mid(k \leqslant j) \&(j \leqslant l)\right\} .
$$

Moreover, $\overrightarrow{m, \infty} \triangleq\left\{j \in \mathbb{N}_{o} \mid m \leqslant j\right\} \quad \forall m \in \mathbb{N}_{o}$. If $H$ is a set and $k \in \mathbb{N}$, then (as usual), $H^{k}$ is used instead of $H^{\overline{1, k}}$; in addition, we assume that elements of $\mathbb{N}$ (natural numbers) are not sets. We recall that $H^{\mathbb{N}}$ is the set of all sequences in $H$; evidently, a family can be used as $H$. In this connection, we note that, for every set $\mathbb{H}$, a sequence $\left(H_{i}\right)_{i \in \mathbb{N}} \in \mathcal{P}(\mathbb{H})^{\mathbb{N}}$, and $H \in \mathcal{P}(\mathbb{H})$,

$$
\left(\left(H_{i}\right)_{i \in \mathbb{N}} \downarrow H\right) \stackrel{\text { def }}{\Longleftrightarrow}\left(\left(H=\bigcap_{i \in \mathbb{N}} H_{i}\right) \&\left(H_{j+1} \subset H_{j} \quad \forall j \in \mathbb{N}\right)\right) .
$$

Recall that, for every TS $(X, \tau)$, the sets $Y \in \sigma_{X}^{o}(\tau)$ are called the Borel sets. Measures defined on $\sigma_{X}^{o}(\tau)$ are also called Borel measures.

If $(E, \mathcal{E})$ is a (standard) measurable space $(E$ is a set and $\mathcal{E}$ is a $\sigma$-algebra of subsets of $E$ ), then, by $(\sigma-\mathrm{add})_{+}[\mathcal{E}]$, we denote the set of all nonnegative real-valued ( $\sigma$-additive) measures on $\mathcal{E}$. Certainly, elements of $(\sigma-\text { add })_{+}[\mathcal{E}]$ are nonnegative real-valued functions defined on $\mathcal{E}$. We note that, for every set $X, \mathcal{X} \in \mathcal{P}^{\prime}(\mathcal{P}(X)), \mu \in(\sigma-\operatorname{add})_{+}\left[\sigma_{X}^{o}(\mathcal{X})\right]$, and $Y \in \sigma_{X}^{o}(\mathcal{X})$, the measurerestriction

$$
\left(\mu \mid \sigma_{Y}^{o}\left(\left.\mathcal{X}\right|_{Y}\right)\right)=\left(\mu\left|\sigma_{X}^{o}(\mathcal{X})\right|_{Y}\right) \in(\sigma-\operatorname{add})_{+}\left[\sigma_{Y}^{o}\left(\left.\mathcal{X}\right|_{Y}\right)\right]
$$

is well-defined (see (1.2) and (1.3)). 


\section{Guidance problem (informative setting)}

In the following, we fix $n \in \mathbb{N}$ as the dimension of the phase space of the system

$$
\dot{x}=f(t, x, u, v), \quad u \in P, v \in Q,
$$

considered on a finite time interval $T \triangleq\left[t_{o}, \vartheta_{o}\right]$, where $t_{o} \in \mathbb{R}$ and $\left.\vartheta_{o} \in\right] t_{o}, \infty[$, or on intervals $\left[t, \vartheta_{o}\right]$ for $t \in T$. Assume that $P$ and $Q$ are nonempty finite-dimensional compact sets; $P \subset \mathbb{R}^{p}$ and $Q \subset \mathbb{R}^{q}$, where $p \in \mathbb{N}$ and $q \in \mathbb{N}$. In addition,

$$
f: T \times \mathbb{R}^{n} \times P \times Q \longrightarrow \mathbb{R}^{n} .
$$

In the following, we equip all sets $\mathbb{R}^{k}, k \in \mathbb{N}$, with the topology of coordinatewise convergence. We assume that $f$ in (2.2) is a vector function that is continuous with respect to the all totality of its variables. Below, we will impose additional conditions on (2.1) and (2.2). Normally, the program (open-loop, non-feedback) controls of players I and II are identified with Borel functions operating from $\left[t, \vartheta_{o}\right]$ into $P$ and $Q$, respectively; here, $t \in T$. However, below, we are going to introduce generalized controls defined as strategic Borel measures corresponding to the sets $\left[t, \vartheta_{o}\right] \times P,\left[t, \vartheta_{o}\right] \times$ $Q$, and $\left[t, \vartheta_{o}\right] \times P \times Q$.

The set $T \times \mathbb{R}^{n}$ is considered as the set of all positions. In this set, we fix the target set and the set that defines phase constraints. For this pair of sets, by the alternative theorem of N.N. Krasovskii and A.I. Subbotin, the corresponding (alternative) partition is defined. More exactly, the set of positions, for which the guidance problem can be successfully solved (solvability set) is determined, the complement of which with respect to the set that defines the phase constraints is the solvability set of the evasion problem. In addition, we now consider the solvability in the corresponding class of positional strategies.

So, we fix two sets $M$ and $N$ that are assumed (hereinafter) to be closed in $T \times \mathbb{R}^{n}$ with the usual topology of coordinatewise convergence (in the following, some conditions will be relaxed; however, we presently adhere to the assumptions of $[2,3])$. We consider counterstrategies

$$
U: T \times \mathbb{R}^{n} \times Q \longrightarrow P
$$

and, in particular, positional strategies

$$
U: T \times \mathbb{R}^{n} \longrightarrow P
$$

as admissible control procedures for the player I, interested in the solution of the guidance problem with the target set $M$ and phase constraints defined by $N$. The second player can use positional strategies

$$
V: T \times \mathbb{R}^{n} \longrightarrow Q
$$

So, $M$ is the target set of player I, and $N$ is the set that defines the corresponding phase constraints; $M \subset T \times \mathbb{R}^{n}$ and $N \subset T \times \mathbb{R}^{n}$.

If $U$ is a counterstrategy $(2.3)$ and $\left(t_{*}, x_{*}\right) \in T \times \mathbb{R}^{n}$, then, by virtue of constructions of [12, p. 239], there exists the bundle $\mathcal{X}_{I}\left(t_{*}, x_{*}, U\right)$ of all trajectories of system (2.1), generated by $U$ from the position $\left(t_{*}, x_{*}\right)$ (in addition, we assume that $U(t, x, \cdot)$ is a Borel function for all $t \in T$ and $\left.x \in \mathbb{R}^{n}\right)$. Trajectories of the bundle $\mathcal{X}_{I}\left(t_{*}, x_{*}, U\right)$ are realized as uniform limits of stepwise movements formed in discrete schemes under unbounded refinement of partitions for the interval $\left[t_{*}, \vartheta_{o}\right]$. It is easy to see that $\mathcal{X}_{I}\left(t_{*}, x_{*}, U\right)$ is defined for every strategy (2.4). In this notation, a position $\left(t_{*}, x_{*}\right) \in N$ is called successful for player I if, for some counterstrategy $U$ (2.3), we have

$$
\forall x(\cdot) \in \mathcal{X}_{I}\left(t_{*}, x_{*}, U\right) \exists \vartheta \in\left[t_{*}, \vartheta_{o}\right]:((\vartheta, x(\vartheta)) \in M) \&\left(( t , x ( t ) ) \in N \quad \forall t \in \left[t_{*}, \vartheta[)\right.\right.
$$


(Certainly, here, we can restrict ourselves to using only strategies (2.4)). Let $W(M, N)$ be the set of all positions successful for player I; in addition, $W(M, N) \subset N$. By the alternative theorem of N.N. Krasovskii and A.I. Subbotin, $N \backslash W(M, N)$ is the set of all positions $\left(t^{*}, x^{*}\right) \in N$ for which there exists a positional strategy $V(2.5)$ with the following property of the bundle $\mathcal{X}_{I I}\left(t^{*}, x^{*}, V\right)$ of trajectories generated $[2,3,12]$ by $V$ from $\left(t^{*}, x^{*}\right)$ :

$$
\forall x(\cdot) \in \mathcal{X}_{I I}\left(t^{*}, x^{*}, V\right) \quad \forall \vartheta \in\left[t^{*}, \vartheta_{o}\right]:((\vartheta, x(\vartheta)) \in M) \Longrightarrow\left(\exists t \in \left[t^{*}, \vartheta[:(t, x(t)) \notin N) .\right.\right.
$$

So, $W(M, N)$ and $N \backslash W(M, N)$ realize the alternative partition of $N$ in the sense of the theorem of N.N. Krasovskii and A.I. Subbotin. If

$$
\min _{u \in P} \max _{v \in Q} s^{\prime} f(t, x, u, v)=\max _{v \in Q} \min _{u \in P} s^{\prime} f(t, x, u, v) \quad \forall s \in \mathbb{R}^{n} \quad \forall t \in T \quad \forall x \in \mathbb{R}^{n},
$$

then $W(M, N)$ is the set of all positions $\left(t_{*}, x_{*}\right) \in N$ for which there exists a strategy $U(2.4)$ realizing (2.6). A more detailed and complete information on the above-mentioned alternative partition may be found in $[2,3]$. In the present study, we consider a question connected with the properties of $W(M, N)$ and procedures realizing the construction of $W(M, N)$. In addition, we use set-valued generalized quasistrategies considered in $[4-6,9,12]$. We note the connection between the solvability in the class of positional strategies and solvability in the class of quasistrategies established in [13] (moreover, see [9, Section 8]).

\section{Generalized controls and trajectories}

We note that, under traditional constraints [2,12], with respect to $f(2.2)$, the bundle of ordinary trajectories of system (2.1) can be a noncompact set in the topology of uniform convergence. Therefore (as in [12, ch. IV]), we introduce a natural extension of the control space in the class of strategic measures (this construction is equivalent to the extension in the class of measure-valued functions used in $[2,14])$.

If $t \in T$, we obtain finite-dimensional compact sets $\left[t, \vartheta_{o}\right], Z_{t} \triangleq\left[t, \vartheta_{o}\right] \times Q$, and $\Omega_{t} \triangleq\left[t, \vartheta_{o}\right] \times$ $P \times Q$. As usual, they are considered together with their natural topologies of coordinatewise convergence. Thus, for $t \in T$, we obtain $\sigma$-algebras $\mathcal{T}_{t}, \mathcal{D}_{t}$, and $\mathcal{C}_{t}$ of Borel sets for $\left[t, \vartheta_{o}\right], Z_{t}$, and $\Omega_{t}$, respectively; thus, we obtain the measurable spaces

$$
\left(\left[t, \vartheta_{o}\right], \mathcal{T}_{t}\right),\left(Z_{t}, \mathcal{D}_{t}\right),\left(\Omega_{t}, \mathcal{C}_{t}\right) .
$$

In (3.1), we have three Borel spaces. Now, we note the required concrete variants of (1.2) and (1.3). Namely, for $t \in T$ and $\theta \in\left[t, \vartheta_{o}\right]$, we obtain

$$
\begin{gathered}
\mathcal{T}_{\theta}=\left.\mathcal{T}_{t}\right|_{\left[\theta, \vartheta_{o}\right]}=\left\{\Gamma \in \mathcal{T}_{t} \mid \Gamma \subset\left[\theta, \vartheta_{o}\right]\right\}, \\
\mathcal{D}_{\theta}=\left.\mathcal{D}_{t}\right|_{Z_{\theta}}=\left\{D \in \mathcal{D}_{t} \mid D \subset Z_{\theta}\right\}, \\
\mathcal{C}_{\theta}=\left.\mathcal{C}_{t}\right|_{\Omega_{\theta}}=\left\{C \in \mathcal{C}_{t} \mid C \subset \Omega_{\theta}\right\}
\end{gathered}
$$

moreover, we introduce $\sigma$-algebras

$\left.\mathcal{C}_{t}^{(\theta)} \triangleq \mathcal{C}_{t}\right|_{[t, \theta[\times P \times Q}=\left\{H \in \mathcal{C}_{t} \mid H \subset\left[t, \theta[\times P \times Q\}\right.\right.$ and $\left.\mathcal{D}_{t}^{(\theta)} \triangleq \mathcal{D}_{t}\right|_{[t, \theta[\times Q}=\left\{D \in \mathcal{D}_{t} \mid D \subset[t, \theta[\times Q\}\right.$

of subsets of $\left[t, \theta\left[\times P \times Q \in \mathcal{C}_{t}\right.\right.$ and $\left[t, \theta\left[\times Q \in \mathcal{D}_{t}\right.\right.$ respectively.

In the form of

$$
\left(\left[t , \theta [ \times P \times Q , \mathcal { C } _ { t } ^ { ( \theta ) } ) \text { and } \left(\left[t, \theta\left[\times Q, \mathcal{D}_{t}^{(\theta)}\right),\right.\right.\right.\right.
$$

we obtain two subspaces of $\left(\Omega_{t}, \mathcal{C}_{t}\right)$ and $\left(Z_{t}, \mathcal{D}_{t}\right)$, respectively. For all $t \in T$, the following important properties hold:

$$
\left(\Gamma \times P \times Q \in \mathcal{C}_{t} \quad \forall \Gamma \in \mathcal{T}_{t}\right) \&\left(D \underline{\times} P \triangleq\left\{(t, u, v) \in \Omega_{t} \mid(t, v) \in D\right\} \in \mathcal{C}_{t} \quad \forall D \in \mathcal{D}_{t}\right) ;
$$


using (3.4), we assume that

$$
\begin{gathered}
\left(\mathcal{H}_{t} \triangleq\left\{\eta \in(\sigma-\text { add })_{+}\left[\mathcal{C}_{t}\right] \mid \eta(\Gamma \times P \times Q)=\lambda(\Gamma) \quad \forall \Gamma \in \mathcal{T}_{t}\right\}\right) \& \\
\&\left(\mathcal{E}_{t} \triangleq\left\{\nu \in(\sigma-\text { add })_{+}\left[\mathcal{D}_{t}\right] \mid \nu(\Gamma \times Q)=\lambda(\Gamma) \quad \forall \Gamma \in \mathcal{T}_{t}\right\}\right),
\end{gathered}
$$

where $\lambda$ is the restriction of the Lebesgue measure to $\mathcal{T}_{t_{o}}$ and (moreover)

$$
\Pi_{t}(\nu) \triangleq\left\{\eta \in \mathcal{H}_{t} \mid \eta(D \underline{\times} P)=\nu(D) \quad \forall D \in \mathcal{D}_{t}\right\} \quad \forall \nu \in \mathcal{E}_{t}
$$

In addition, the measures from $\mathcal{H}_{t}$ play the role of pairs $(u(\cdot), v(\cdot))$, where

$$
u(\cdot):\left[t, \vartheta_{o}\right] \longrightarrow P, v(\cdot):\left[t, \vartheta_{o}\right] \longrightarrow Q
$$

are Borel functions. Measures of $\mathcal{E}_{t}$ are generalized analogs of Borel functions $v(\cdot)$ (see (3.7)). Finally, measures $\eta \in \Pi_{t}(\nu)$ correspond to pairs $(u(\cdot), \bar{v}(\cdot))$, where $\bar{v}(\cdot)$ is fixed.

We note that, for $t \in T$ and $\theta \in\left[t, \vartheta_{o}\right]$,

$$
\left(\mathcal{H}_{\theta}=\left\{\left(\eta \mid \mathcal{C}_{\theta}\right): \eta \in \mathcal{H}_{t}\right\}\right) \&\left(\mathcal{E}_{\theta}=\left\{\left(\nu \mid \mathcal{D}_{\theta}\right): \nu \in \mathcal{E}_{t}\right\}\right)
$$

In (3.8), we obtain the restriction properties for generalized controls (we consider the measures from $\mathcal{H}_{\tau}$ and $\mathcal{E}_{\tau}$, where $\tau \in T$, as generalized controls). Moreover, we note the sewing properties: if $t \in T$ and $\theta \in\left[t, \vartheta_{o}\right]$, then

$$
\begin{gathered}
\left(\eta _ { 1 } \perp \eta _ { 2 } \triangleq \left(\eta_{1}\left(H \cap\left([t, \theta[\times P \times Q))+\eta_{2}\left(H \cap \Omega_{\theta}\right)\right)_{H \in \mathcal{C}_{t}} \in \mathcal{H}_{t} \quad \forall \eta_{1} \in \mathcal{H}_{t} \quad \forall \eta_{2} \in \mathcal{H}_{\theta}\right) \&\right.\right. \\
\&\left(\nu _ { 1 } \bowtie \nu _ { 2 } \triangleq \left(\nu_{1}\left(D \cap\left([t, \theta[\times Q))+\nu_{2}\left(D \cap Z_{\theta}\right)\right)_{D \in \mathcal{D}_{t}} \in \mathcal{E}_{t} \quad \forall \nu_{1} \in \mathcal{E}_{t} \quad \forall \nu_{2} \in \mathcal{E}_{\theta}\right) .\right.\right.
\end{gathered}
$$

Now, using (3.3), we assume that, for all $t \in T, \theta \in\left[t, \vartheta_{o}\right]$, and $\mathbb{H} \in \mathcal{P}\left(\mathcal{H}_{t}\right)$,

$$
[\mathbb{H} \mid t, \theta) \triangleq\left\{\left(\eta \mid \mathcal{C}_{t}^{(\theta)}\right): \eta \in \mathbb{H}\right\} .
$$

In the following, for $t \in T$, we use the sets $C\left(\left[t, \vartheta_{o}\right]\right), C\left(\Omega_{t}\right)$, and $C\left(Z_{t}\right)$ of all continuous real-valued functions defined on $\left[t, \vartheta_{o}\right], \Omega_{t}$, and $Z_{t}$, respectively. Certainly, $C\left(\left[t, \vartheta_{o}\right]\right), C\left(\Omega_{t}\right)$, and $C\left(Z_{t}\right)$, with corresponding norms of uniform convergence, are Banach spaces. We denote by $C^{*}\left(\Omega_{t}\right)$ and $C^{*}\left(Z_{t}\right)$ the spaces that are topologically conjugate to Banach spaces $C\left(\Omega_{t}\right)$ and $C\left(Z_{t}\right)$, respectively. Using the known Riesz theorem, we can regard $\mathcal{H}_{t}$ and $\mathcal{E}_{t}$ as subsets of $C^{*}\left(\Omega_{t}\right)$ and $C^{*}\left(Z_{t}\right)$, respectively (we keep in mind the corresponding embedding of $(\sigma-\operatorname{add})_{+}\left[\mathcal{C}_{t}\right]$ and $(\sigma-\operatorname{add})_{+}\left[\mathcal{D}_{t}\right]$ into $C_{+}^{*}\left(\Omega_{t}\right)$ and $C_{+}^{*}\left(Z_{t}\right)$, where $C_{+}^{*}\left(\Omega_{t}\right)$ and $C_{+}^{*}\left(Z_{t}\right)$ are the cones of nonnegative elements of $C^{*}\left(\Omega_{t}\right)$ and $C^{*}\left(Z_{t}\right)$, respectively). Therefore, we equip $\mathcal{H}_{t}$ and $\mathcal{E}_{t}$ with the corresponding relative ${ }^{*}$-weak topologies. In addition, the base of the relative ${ }^{*}$-weak topology of $\mathcal{H}_{t}$ is the family of all sets

$$
\left.\left\{\tilde{\eta} \in \mathcal{H}_{t}|| \int_{\Omega_{t}} h d \eta-\int_{\Omega_{t}} h d \tilde{\eta} \mid<\varepsilon \forall h \in K\right\}, \eta \in \mathcal{H}_{t}, K \in \operatorname{Fin}\left(C\left(\Omega_{t}\right)\right), \varepsilon \in\right] 0, \infty[.
$$

Analogously, the base of the relative ${ }^{*}$-weak topology of $\mathcal{E}_{t}$ is the family of all sets

$$
\left.\left\{\tilde{\nu} \in \mathcal{E}_{t}|| \int_{Z_{t}} h d \nu-\int_{Z_{t}} h d \tilde{\nu} \mid<\varepsilon \forall h \in K\right\}, \nu \in \mathcal{E}_{t}, K \in \operatorname{Fin}\left(C\left(Z_{t}\right)\right), \varepsilon \in\right] 0, \infty[.
$$

Recall that $C\left(\Omega_{t}\right)$ and $C\left(Z_{t}\right)$, supplemented with the corresponding metrics of uniform convergence, are separable spaces. Therefore, the above-mentioned relative ${ }^{*}$-weak topologies of $\mathcal{H}_{t}$ and $\mathcal{E}_{t}$ are metrizable (we recall that $\mathcal{H}_{t}$ and $\mathcal{E}_{t}$ are strongly bounded). As a corollary, we find that, for all 
subsets of $\mathcal{H}_{t}$ and $\mathcal{E}_{t}$, the closure property is equivalent to the sequential closure property (in fact, the closures of subsets of $\mathcal{H}_{t}$ and $\mathcal{E}_{t}$ coincide with their sequential closures). Analogously, for $\mathcal{H}_{t}$ and $\mathcal{E}_{t}$, compactness and sequential compactness are identified (we keep in mind the above-mentioned relative ${ }^{*}$-weak topologies). Using the Alaoglu theorem, we find that $\mathcal{H}_{t}$ and $\mathcal{E}_{t}$ are (sequentially) ${ }_{-}$ weak compact. Moreover, for all $\nu \in \mathcal{E}_{t}$, the set $\Pi_{t}(\nu)(3.6)$ is a (sequentially) ${ }^{*}$-weak compact set. A more detailed information about the properties of $\mathcal{H}_{t}, \mathcal{E}_{t}$, and $\Pi_{t}(\nu), \nu \in \mathcal{E}_{t}$, is given in [12, ch. IV].

For $t_{*} \in T$, we denote by $C_{n}\left(\left[t_{*}, \vartheta_{o}\right]\right)$ the set of all continuous functions from $\left[t_{*}, \vartheta_{o}\right]$ into $\mathbb{R}^{n}$ (recall that $\mathbb{R}^{n}$ is equipped with the topology of coordinatewise convergence). If $x(\cdot)=(x(t))_{t \in\left[t_{*}, \vartheta_{o}\right]} \in$ $C_{n}\left(\left[t_{*}, \vartheta_{o}\right]\right)$, then

$$
(t, u, v) \longmapsto f(t, x(t), u, v): \Omega_{t} \longrightarrow \mathbb{R}^{n}
$$

is a continuous mapping with components from $C\left(\Omega_{t_{*}}\right)$; therefore,

$$
\int_{\left[t_{*}, \theta[\times P \times Q\right.} f(t, x(t), u, v) \eta(d(t, u, v)) \in \mathbb{R}^{n}
$$

is defined componentwise for $\eta \in \mathcal{H}_{t_{*}}$ and $\theta \in\left[t_{*}, \vartheta_{o}\right]$ (we note that integral (3.11) can be defined by a most simple scheme of [15, ch. 3]). Using (3.11), we introduce integral funnel: if $\left(t_{*}, x_{*}\right) \in T \times \mathbb{R}^{n}$ and $\eta \in \mathcal{H}_{t_{*}}$, we assume that

$$
\Phi\left(t_{*}, x_{*}, \eta\right) \triangleq\left\{x(\cdot) \in C_{n}\left(\left[t_{*}, \vartheta_{o}\right]\right) \mid x(t)=x_{*}+\int_{\left[t_{*} . t[\times P \times Q\right.} f(\xi, x(\xi), u, v) \eta(d(\xi, u, v)) \forall t \in\left[t_{*}, \vartheta_{o}\right]\right\} .
$$

In the following, we assume that

$$
\forall\left(t_{*}, x_{*}\right) \in T \times \mathbb{R}^{n} \quad \forall \eta \in \mathcal{H}_{t_{*}} \exists ! x^{*}(\cdot) \in C_{n}\left(\left[t_{*}, \vartheta_{o}\right]\right): \Phi\left(t_{*}, x_{*}, \eta\right)=\left\{x^{*}(\cdot)\right\}
$$

((3.13) is a condition of generalized uniqueness; see [10]). Using (3.13), we assume that, for all $\left(t_{*}, x_{*}\right) \in T \times \mathbb{R}^{n}$ and $\eta \in \mathcal{H}_{t_{*}}$, the vector function

$$
\varphi\left(\cdot, t_{*}, x_{*}, \eta\right)=\left(\varphi\left(t, t_{*}, x_{*}, \eta\right)\right)_{t \in\left[t_{*}, \vartheta_{o}\right]} \in C_{n}\left(\left[t_{*}, \vartheta_{o}\right]\right)
$$

satisfies the following condition

$$
\Phi\left(t_{*}, x_{*}, \eta\right)=\left\{\varphi\left(\cdot, t_{*}, x_{*}, \eta\right)\right\} .
$$

Thus, in (3.14) and (3.15), generalized trajectories of system (2.1) are introduced. From (3.15), using most simple statements of measure theory (see, for example, [15, (4.4.28)]), one can establish that

(1) $\forall\left(t_{*}, x_{*}\right) \in T \times \mathbb{R}^{N} \forall \eta_{1} \in \mathcal{H}_{t_{*}} \forall \eta_{2} \in \mathcal{H}_{t_{*}} \forall \theta \in\left[t_{*}, \vartheta_{o}\right]$

$$
\left(\left(\eta_{1} \mid \mathcal{C}_{t_{*}}^{(\theta)}\right)=\left(\eta_{2} \mid \mathcal{C}_{t_{*}}^{(\theta)}\right)\right) \Longrightarrow\left(\varphi\left(t, t_{*}, x_{*}, \eta_{1}\right)=\varphi\left(t, t_{*}, x_{*}, \eta_{2}\right) \quad \forall t \in\left[t_{*}, \theta\right]\right) ;
$$

(2) $\forall\left(t_{*}, x_{*}\right) \in T \times \mathbb{R}^{n} \forall \eta_{1} \in \mathcal{H}_{t_{*}} \forall \theta \in\left[t_{*}, \vartheta_{o}\right] \quad \forall \eta_{2} \in \mathcal{H}_{\theta}$

$$
\begin{gathered}
\left(\varphi\left(t, t_{*}, x_{*}, \eta_{1}\right)=\varphi\left(t, t_{*}, x_{*}, \eta_{1} \perp \eta_{2}\right) \quad \forall t \in\left[t_{*}, \theta\right]\right) \& \\
\&\left(\varphi\left(t, t_{*}, x_{*}, \eta_{1} \perp \eta_{2}\right)=\varphi\left(t, \theta, \varphi\left(\theta, t_{*}, x_{*}, \eta_{1}\right), \eta_{2}\right) \quad \forall t \in\left[\theta, \vartheta_{o}\right]\right) .
\end{gathered}
$$

Clearly, (1) and (2) realize an obvious analog of the semigroup property: if $\left(t_{*}, x_{*}\right) \in T \times \mathbb{R}^{n}, \eta \in$ $\mathcal{H}_{t_{*}}$, and $\theta \in\left[t_{*}, \vartheta_{o}\right]$, then $\left(\eta \mid \mathcal{C}_{\theta}\right) \in \mathcal{H}_{\theta}$ and $\varphi\left(t, t_{*}, x_{*}, \eta\right)=\varphi\left(t, \theta, \varphi\left(\theta, t_{*}, x_{*}, \eta\right),\left(\eta \mid \mathcal{C}_{\theta}\right)\right) \quad \forall t \in\left[\theta, \vartheta_{o}\right]$ (it is easy to see that this property follows from (3.7), (3.9), and (2)).

In the following, let $\|\cdot\|$ be the Euclidean norm in $\mathbb{R}^{n}$ by definition. Assume that

$$
\mathbb{B}_{n}(a) \triangleq\left\{x \in \mathbb{R}^{n} \mid\|x\| \leqslant a\right\} \quad \forall a \in[0, \infty[.
$$


We assume further that $\forall \alpha \in[0, \infty[\exists \beta \in[0, \infty[$ :

$$
\varphi\left(t, t_{*}, x_{*}, \eta\right) \in \mathbb{B}_{n}(\beta) \quad \forall t_{*} \in T \quad \forall x_{*} \in \mathbb{B}_{n}(\alpha) \forall \eta \in \mathcal{H}_{t_{*}} \forall t \in\left[t_{*}, \vartheta_{o}\right] .
$$

Returning to (3.5), let us agree on the following notational convention: the symbol $\rightarrow$ denotes the convergence in the sense of (relative) ${ }^{*}$-weak topologies. So, if $t_{*} \in T,\left(\eta_{i}\right)_{i \in \mathbb{N}}: \mathbb{N} \rightarrow \mathcal{H}_{t_{*}}$, and $\eta \in \mathcal{H}_{t_{*}}$, then

$$
\left(\left(\eta_{i}\right)_{i \in \mathbb{N}} \rightarrow \eta\right) \stackrel{\text { def }}{\Longleftrightarrow}\left(\left(\int_{\Omega_{t_{*}}} h d \eta_{i}\right)_{i \in \mathbb{N}} \longrightarrow \int_{\Omega_{t_{*}}} h d \eta \forall h \in C\left(\Omega_{t_{*}}\right)\right) .
$$

Analogously, if $t_{*} \in T,\left(\nu_{i}\right)_{i \in \mathbb{N}}: \mathbb{N} \rightarrow \mathcal{E}_{t_{*}}$, and $\nu \in \mathcal{E}_{t_{*}}$, then

$$
\left(\left(\nu_{i}\right)_{i \in \mathbb{N}} \rightarrow \nu\right) \stackrel{\text { def }}{\Longleftrightarrow}\left(\left(\int_{Z_{t_{*}}} h d \nu_{i}\right)_{i \in \mathbb{N}} \longrightarrow \int_{Z_{t_{*}}} h d \nu \quad \forall h \in C\left(Z_{t_{*}}\right)\right) .
$$

Of course, in (3.17) and (3.18), we have the usual *-weak convergence. We note the following general property (here and below, $\rightrightarrows$ denotes the uniform convergence): if $t_{*} \in T, x_{*} \in \mathbb{R}^{n}$, and $\eta \in \mathcal{H}_{t_{*}}$,

$$
\left(\eta_{i}\right)_{i \in \mathbb{N}}: \mathbb{N} \longrightarrow \mathcal{H}_{t_{*}}, \text { and }\left(x_{*}^{(i)}\right)_{i \in \mathbb{N}}: \mathbb{N} \longrightarrow \mathbb{R}^{n},
$$

then the following implication is true:

$$
\left(\left(\left(\eta_{i}\right)_{i \in \mathbb{N}} \rightarrow \eta\right) \&\left(\left(x_{*}^{(i)}\right)_{i \in \mathbb{N}} \rightarrow x_{*}\right)\right) \Rightarrow\left(\left(\varphi\left(\cdot, t_{*}, x_{*}^{(i)}, \eta_{i}\right)\right)_{i \in \mathbb{N}} \rightrightarrows \varphi\left(\cdot, t_{*}, x_{*}, \eta\right)\right) .
$$

In (3.19), we obtain an important property of continuity. This property (in combination with the compactness of $\mathcal{H}_{t_{*}}$ ) defines new essential possibilities in the scheme employing the generalized controls.

\section{Operator of program absorption: general properties}

We equip $T \times \mathbb{R}^{n}$ with the natural topology $\mathbf{t}$ of coordinatewise convergence. This topology is generated by the metric $\rho$ defined as

$$
\left(\left(t_{1}, x_{1}\right),\left(t_{2}, x_{2}\right)\right) \longmapsto \sup \left(\left\{\left|t_{1}-t_{2}\right| ;\left\|x_{1}-x_{2}\right\|\right\}\right):\left(T \times \mathbb{R}^{n}\right) \times\left(T \times \mathbb{R}^{n}\right) \longrightarrow[0, \infty[.
$$

By $\mathcal{F}$, we denote the family of all subsets of $T \times \mathbb{R}^{n}$ that are closed in TS $\left(T \times \mathbb{R}^{n}, \mathbf{t}\right)$. This TS can be considered as a natural product $[16$, ch. 2$]$ of $T$ with the natural $|\cdot|$-topology and $\left(\mathbb{R}^{n}, \tau_{\mathbb{R}}^{(n)}\right)$, where $\tau_{\mathbb{R}}^{(n)}$ is the ordinary topology of coordinate-wise convergence in $\mathbb{R}^{n}\left(\tau_{\mathbb{R}}^{(n)}\right.$ generated by the norm $\left.\|\cdot\|\right)$. Assume that $\tau_{\partial} \triangleq \mathcal{P}(T)$. Then, $\left(T, \tau_{\partial}\right)$ is a discrete TS. We consider the natural topology $\tau_{\partial} \otimes \tau_{\mathbb{R}}^{(n)}$ corresponding to the direct product $[16$, ch. 2$]$ of the TSs $\left(T, \tau_{\partial}\right)$ and $\left(\mathbb{R}^{n}, \tau_{\mathbb{R}}^{(n)}\right)$. So, we obtain a "semidiscrete" TS. Let $\mathfrak{F}$ be the family of all subsets of $T \times \mathbb{R}^{n}$ closed in the TS $\left(T \times \mathbb{R}^{n}, \tau_{\partial} \otimes \tau_{\mathbb{R}}^{(n)}\right)$. For representation of $\mathfrak{F}$, we introduce

$$
H\langle t\rangle \triangleq\left\{x \in \mathbb{R}^{n} \mid(t, x) \in H\right\} \quad \forall H \in \mathcal{P}\left(T \times \mathbb{R}^{n}\right) \quad \forall t \in T .
$$

Then, $\mathfrak{F}=\left\{F \in \mathcal{P}\left(T \times \mathbb{R}^{n}\right) \mid F\langle t\rangle \in \mathbf{F} \quad \forall t \in T\right\}$, where $\mathbf{F}$ is the family of all subsets of $\mathbb{R}^{n}$ closed in $\left(\mathbb{R}^{n}, \tau_{\mathbb{R}}^{(n)}\right)$. We note that

$$
\left(\mathbf{t} \subset \tau_{\partial} \otimes \tau_{\mathbb{R}}^{(n)}\right) \&(\mathcal{F} \subset \mathfrak{F}) .
$$

Now, we introduce the mappings operating in $\mathcal{P}\left(T \times \mathbb{R}^{n}\right)$. Each such mapping is connected with a fixed subset of $T \times \mathbb{R}^{n}$. Namely, if $M \in \mathcal{P}\left(T \times \mathbb{R}^{n}\right)$, then

$$
\mathbb{A}[M]: \mathcal{P}\left(T \times \mathbb{R}^{n}\right) \longrightarrow \mathcal{P}\left(T \times \mathbb{R}^{n}\right)
$$


is defined by the rule

$$
\begin{gathered}
\mathbb{A}[M](S) \triangleq\left\{(t, x) \in S \mid \forall \nu \in \mathcal{E}_{t} \exists \eta \in \Pi_{t}(\nu) \exists \vartheta \in\left[t, \vartheta_{o}\right]:\right. \\
((\vartheta, \varphi(\vartheta, t, x, \eta)) \in M) \&\left((\xi, \varphi(\xi, t, x, \eta)) \in S \quad \forall \xi \in[t, \vartheta[)\} \quad \forall S \in \mathcal{P}\left(T \times \mathbb{R}^{n}\right) ;\right.
\end{gathered}
$$

in addition, $M \cap S \subset \mathbb{A}[M](S)$ for $S \in \mathcal{P}\left(T \times \mathbb{R}^{n}\right)$, and

$$
\begin{gathered}
\mathbb{A}[M](F) \triangleq\left\{(t, x) \in F \mid \forall \nu \in \mathcal{E}_{t} \exists \eta \in \Pi_{t}(\nu) \exists \vartheta \in\left[t, \vartheta_{o}\right]:\right. \\
((\vartheta, \varphi(\vartheta, t, x, \eta)) \in M) \&((\xi, \varphi(\xi, t, x, \eta)) \in F \quad \forall \xi \in[t, \vartheta])\} \quad \forall F \in \mathcal{F} .
\end{gathered}
$$

From (4.5), the next obvious property follows: if $M_{1} \in \mathcal{P}\left(T \times \mathbb{R}^{n}\right), S_{1} \in \mathcal{P}\left(T \times \mathbb{R}^{n}\right), M_{2} \in \mathcal{P}\left(T \times \mathbb{R}^{n}\right)$, and $S_{2} \in \mathcal{P}\left(T \times \mathbb{R}^{n}\right)$, then

$$
\left(\left(M_{1} \subset M_{2}\right) \&\left(S_{1} \subset S_{2}\right)\right) \Longrightarrow\left(\mathbb{A}\left[M_{1}\right]\left(S_{1}\right) \subset \mathbb{A}\left[M_{2}\right]\left(S_{2}\right)\right) .
$$

In particular, every operator (4.4), (4.5) is an isotone mapping.

Proposition 1. If $M \in \mathcal{F}$, then $\mathfrak{F}$ is an invariant subspace of the operator $\mathbb{A}[M]$,

$$
\mathbb{A}[M](F) \in \mathfrak{F} \quad \forall F \in \mathfrak{F} .
$$

The corresponding proof easily follows from (3.19) and the compactness property of sets (3.6).

Proposition 2. If $\left(M_{i}\right)_{i \in \mathbb{N}}: \mathbb{N} \rightarrow \mathcal{F},\left(F_{i}\right)_{i \in \mathbb{N}}: \mathbb{N} \rightarrow \mathfrak{F}, M \in \mathcal{P}\left(T \times \mathbb{R}^{n}\right), F \in \mathcal{P}\left(T \times \mathbb{R}^{n}\right)$, and

$$
\left(\left(M_{i}\right)_{i \in \mathbb{N}} \downarrow M\right) \&\left(\left(F_{i}\right)_{i \in \mathbb{N}} \downarrow F\right),
$$

then $M \in \mathcal{F}, F \in \mathfrak{F}$, and $\left(\mathbb{A}\left[M_{i}\right]\left(F_{i}\right)\right)_{i \in \mathbb{N}} \downarrow \mathbb{A}[M](F)$.

The proof uses (3.19) and compactness of sets (3.6).

\section{The method of program iterations}

We follow the traditional requirements: if $X$ is a nonempty set and $\gamma \in X^{X}$, then the sequence

$$
\left(\gamma^{k}\right)_{k \in \mathbb{N}_{o}}: \mathbb{N}_{o} \longrightarrow X^{X}
$$

is defined by the following conditions: 1) $\gamma^{o}(x) \triangleq x \forall x \in X$; 2) $\gamma^{k+1}=\gamma \circ \gamma^{k} \forall k \in \mathbb{N}_{o}$. We realize this requirement for $X=\mathcal{P}\left(T \times \mathbb{R}^{n}\right)$ and $\gamma=\mathbb{A}[M]$, where $M \in \mathcal{P}\left(T \times \mathbb{R}^{n}\right)$. Thus, from (4.4) and (5.1), we obtain

$$
\left(\mathbb{A}[M]^{k}\right)_{k \in \mathbb{N}_{o}}: \mathbb{N}_{o} \longrightarrow \mathcal{P}\left(T \times \mathbb{R}^{n}\right)^{\mathcal{P}\left(T \times \mathbb{R}^{n}\right)} .
$$

In this connection, for $M \in \mathcal{P}\left(T \times \mathbb{R}^{n}\right), N \in \mathcal{P}\left(T \times \mathbb{R}^{n}\right)$, and $k \in \mathbb{N}_{o}$, we assume the following:

$$
W_{k}(M, N) \triangleq \mathbb{A}[M]^{k}(N) .
$$

Thus, we obtain sequences in $\mathcal{P}\left(T \times \mathbb{R}^{n}\right)$. More precisely, for $M \in \mathcal{P}\left(T \times \mathbb{R}^{n}\right)$ and $N \in \mathcal{P}\left(T \times \mathbb{R}^{n}\right)$, we obtain

$$
\left(W_{k}(M, N)\right)_{k \in \mathbb{N}_{o}}: \mathbb{N}_{o} \longrightarrow \mathcal{P}\left(T \times \mathbb{R}^{n}\right)
$$

defined as follows:

$$
\left(W_{o}(M, N)=N\right) \&\left(W_{s+1}(M, N)=\mathbb{A}[M]\left(W_{s}(M, N)\right) \forall s \in \mathbb{N}_{o}\right)
$$


using (5.3) and(5.4), we set

$$
W(M, N) \triangleq \bigcap_{k \in \mathbb{N}_{o}} W_{k}(M, N) .
$$

Naturally, in view of (4.5), (5.4), and (5.5), we obtain (for $M \in \mathcal{P}\left(T \times \mathbb{R}^{n}\right.$ ) and $N \in \mathcal{P}\left(T \times \mathbb{R}^{n}\right)$ ) the following convergence property:

$$
\left(W_{k}(M, N)\right)_{k \in \mathbb{N}} \downarrow W(M, N) .
$$

From (4.5) and (5.4), by induction, we obtain $\forall M_{1} \in \mathcal{P}\left(T \times \mathbb{R}^{n}\right) \quad \forall N_{1} \in \mathcal{P}\left(T \times \mathbb{R}^{n}\right) \quad \forall M_{2} \in$ $\mathcal{P}\left(T \times \mathbb{R}^{n}\right) \quad \forall N_{2} \in \mathcal{P}\left(T \times \mathbb{R}^{n}\right)$

$$
\left(\left(M_{1} \subset M_{2}\right) \&\left(N_{1} \subset N_{2}\right)\right) \Longrightarrow\left(W_{k}\left(M_{1}, N_{1}\right) \subset W_{k}\left(M_{2}, N_{2}\right) \quad \forall k \in \mathbb{N}_{o}\right) .
$$

Consequently, from (5.5) and (5.7), we obtain the following property: $\forall M_{1} \in \mathcal{P}\left(T \times \mathbb{R}^{n}\right) \quad \forall N_{1} \in$ $\mathcal{P}\left(T \times \mathbb{R}^{n}\right) \quad \forall M_{2} \in \mathcal{P}\left(T \times \mathbb{R}^{n}\right) \quad \forall N_{2} \in \mathcal{P}\left(T \times \mathbb{R}^{n}\right)$

$$
\left(\left(M_{1} \subset M_{2}\right) \&\left(N_{1} \subset N_{2}\right)\right) \Longrightarrow\left(W\left(M_{1}, N_{1}\right) \subset W\left(M_{2}, N_{2}\right)\right) .
$$

From Proposition 1 and (5.4), induction yields the following important statement:

$$
W_{s}(M, N) \in \mathfrak{F} \quad \forall M \in \mathcal{F} \quad \forall N \in \mathfrak{F} \quad \forall s \in \mathbb{N}_{o} .
$$

Thus, from (5.5) and (5.9), we obtain

$$
W(M, N) \in \mathfrak{F} \quad \forall M \in \mathcal{F} \quad \forall N \in \mathfrak{F} .
$$

Proposition 3. If $M \in \mathcal{F}$ and $N \in \mathfrak{F}$, then $W(M, N)=\mathbb{A}[M](W(M, N))$.

The proof follows [17] from Proposition 2, (5.4), and (5.6). Now, we note the two obvious properties:

$\left(1^{\prime}\right)$ if $M \in \mathcal{P}\left(T \times \mathbb{R}^{n}\right), N \in \mathcal{P}\left(T \times \mathbb{R}^{n}\right)$, and $H \in \mathcal{P}(N)$, then

$$
(H=\mathbb{A}[M](H)) \Longrightarrow(H \subset W(M, N)) ;
$$

$\left(2^{\prime}\right)$ if $M \in \mathcal{F}, N \in \mathfrak{F}$, and $L \in \mathcal{P}(N)$, then

$$
(W(M, N) \subset L) \Longrightarrow(W(M, N)=W(M, L)) .
$$

The property $\left(1^{\prime}\right)$ follows from $(4.7),(5.4)$, and $(5.5) ;\left(2^{\prime}\right)$ is a simple corollary of $(4.7),(5.4),(5.5)$, and Proposition 3.

Proposition 4. If $\left(M_{i}\right)_{i \in \mathbb{N}}: \mathbb{N} \longrightarrow \mathcal{F},\left(N_{i}\right)_{i \in \mathbb{N}}: \mathbb{N} \longrightarrow \mathfrak{F}, M \in \mathcal{P}\left(T \times \mathbb{R}^{n}\right), N \in \mathcal{P}\left(T \times \mathbb{R}^{n}\right)$, and

$$
\left(\left(M_{i}\right)_{i \in \mathbb{N}} \downarrow M\right) \&\left(\left(N_{i}\right)_{i \in \mathbb{N}} \downarrow N\right),
$$

then $M \in \mathcal{F}, N \in \mathfrak{F}$, and, moreover,

$$
\left(W_{k}\left(M_{i}, N_{i}\right)\right)_{i \in \mathbb{N}} \downarrow W_{k}(M, N) \quad \forall k \in \mathbb{N}_{o} .
$$

The proof follows from Proposition 2 and (5.4) by induction. Using (5.5) and Proposition 4, we obtain the following statement:

Theorem 1. If $\left(M_{i}\right)_{i \in \mathbb{N}}: \mathbb{N} \longrightarrow \mathcal{F},\left(N_{i}\right)_{i \in \mathbb{N}}: \mathbb{N} \longrightarrow \mathfrak{F}, M \in \mathcal{P}\left(T \times \mathbb{R}^{n}\right), N \in \mathcal{P}\left(T \times \mathbb{R}^{n}\right)$, and $\left(\left(M_{i}\right)_{i \in \mathbb{N}} \downarrow M\right) \&\left(\left(N_{i}\right)_{i \in \mathbb{N}} \downarrow N\right)$,

then, $M \in \mathcal{F}, N \in \mathfrak{F}$, and, in addition,

$$
\left(W\left(M_{i}, N_{i}\right)\right)_{i \in \mathbb{N}^{\mathbb{N}}} \downarrow W(M, N) .
$$

From Theorem 1, we see that the mapping

$$
(M, N) \longmapsto W(M, N): \mathcal{F} \times \mathfrak{F} \longrightarrow \mathfrak{F}
$$

is "sequentially continuous" from above (bearing in mind the effect connected with the preservation of monotonicity of convergence of sets). 


\section{One type of invariant subspace of program absorption operator}

In this section, we fix $M \in \mathcal{F}$ and $N \in \mathfrak{F} \backslash\{\emptyset\}$. So, in particular, $N$ is a nonempty subset of $T \times \mathbb{R}^{n}$ (therefore, $N \in \mathcal{P}^{\prime}\left(T \times \mathbb{R}^{n}\right)$ ). Then, the restriction $(\rho \mid N \times N)$ of $\rho$ (4.1) is defined: $(\rho \mid N \times N)$ is the metric of $N$ that has the form

$$
\left(\left(t_{1}, x_{1}\right),\left(t_{2}, x_{2}\right)\right) \longmapsto \sup \left(\left\{\left|t_{1}-t_{2}\right| ;\left\|x_{1}-x_{2}\right\|\right\}\right): N \times N \longrightarrow[0, \infty[.
$$

So, $(\rho \mid N \times N)$ is a mapping (6.1). On the other hand (see (1.1)),

$$
\left.\mathbf{t}\right|_{N}=\{N \cap G: G \in \mathbf{t}\}
$$

is the (relative) topology of $N$ induced by the TS $\left(T \times \mathbb{R}^{n}, \mathbf{t}\right)$. Certainly, $\left(N,\left.\mathbf{t}\right|_{N}\right)$ is a subspace of the latter TS. In addition, $(\rho \mid N \times N)(6.1)$ is the metric of $N$ that generates topology (6.2). Then, the closure of a subset of $N$ is realized in terms of $\rho$ :

$$
\operatorname{cl}\left(S,\left.\mathbf{t}\right|_{N}\right)=\left\{(t, x) \in N \mid \exists\left(\left(t_{i}, x_{i}\right)\right)_{i \in \mathbb{N}} \in S^{\mathbb{N}}:\left(\rho\left(\left(t_{i}, x_{i}\right),(t, x)\right)\right)_{i \in \mathbb{N}} \longrightarrow 0\right\} \quad \forall S \in \mathcal{P}(N) .
$$

Finally, $\left.\mathcal{F}\right|_{N}=\{N \cap F: F \in \mathcal{F}\}$ is the family of all subsets of $N$ closed in the sense of topology (6.2).

Proposition 5. The family $\left.\mathcal{F}\right|_{N}$ is an invariant subspace of $\mathbb{A}[M]:\left.\mathbb{A}[M](F) \in \mathcal{F}\right|_{N} \forall F \in$ $\left.\mathcal{F}\right|_{N}$.

We omit the corresponding proof using (6.1) and (6.2); the basic part of this proof is contained in [17, Section 7]. From Proposition 5, we obtain

$$
\left.W_{k}(M, N) \in \mathcal{F}\right|_{N} \quad \forall k \in \mathbb{N}_{o} .
$$

As a corollary, from (5.5) and (6.4), we obtain

$$
\left.W(M, N) \in \mathcal{F}\right|_{N} .
$$

Thus, $W(M, N)$ is closed in topology (6.2) of the set $N$.

To complete this section, we assume that $N \in \mathcal{F}$ (i.e., we assume that $N$ is closed in $\left(T \times \mathbb{R}^{n}, \mathbf{t}\right)$ ). Then, $\left.\mathcal{F}\right|_{N} \subset \mathcal{F}$. From (6.4), we obtain

$$
W_{k}(M, N) \in \mathcal{F} \quad \forall k \in \mathbb{N}_{o} .
$$

Analogously, from (6.5), we find that, in the considered case,

$$
W(M, N) \in \mathcal{F} .
$$

We note that (6.6) is compatible with basic statements of $[2,3]$ connected with the alternative theorem of N.N. Krasovskii and A.I. Subbotin.

\section{Topological properties of fragments of the set of positional absorption, 1}

We consider a metric space $\left(T \times \mathbb{R}^{n}, \rho\right)$; see (4.1). Set

$$
\rho(z ; H) \triangleq \inf (\{\rho(z, h): h \in H\}) \quad \forall z \in T \times \mathbb{R}^{n} \quad \forall H \in \mathcal{P}^{\prime}\left(T \times \mathbb{R}^{n}\right) .
$$

In particular, from (7.1), we obtain

$$
S_{o}(F, \varepsilon) \triangleq\left\{z \in T \times \mathbb{R}^{n} \mid \rho(z ; F) \leqslant \varepsilon\right\} \in \mathcal{F} \backslash\{\emptyset\} \quad \forall F \in \mathcal{F} \backslash\{\emptyset\} \quad \forall \varepsilon \in[0, \infty[.
$$


Moreover, for $F \in \mathcal{F} \backslash\{\emptyset\}$, we assume

$$
\mathcal{K}[F] \triangleq\left\{K \in(\mathbf{t}-\operatorname{comp})\left[T \times \mathbb{R}^{n}\right] \mid K \cap F \neq \emptyset\right\} .
$$

In addition, for $F \in \mathcal{F} \backslash\{\emptyset\}$ and $K \in \mathcal{K}[F]$, we obtain $K \cap F \in \mathcal{F} \backslash\{\emptyset\}$ and, therefore, for $\varepsilon \in[0, \infty[$, the set $S_{o}(K \cap F, \varepsilon) \in \mathcal{F} \backslash\{\emptyset\}$ is defined; evidently, for $\widetilde{F} \in \mathcal{F}$ such that $F \subset \widetilde{F}$, the property $K \cap \widetilde{F} \in \mathcal{F} \backslash\{\emptyset\}$ holds as well.

Until end of this section, we fix $M \in \mathcal{F}$ and $N \in \mathcal{F}$ with the property $W(M, N) \neq \emptyset$ (obviously, in this case, $M \neq \emptyset$ and $N \neq \emptyset$; see (4.6), (5.4), and (5.5)). Then, by (6.6), $W(M, N) \in \mathcal{F} \backslash\{\emptyset\}$. Therefore, for $K \in \mathcal{K}[W(M, N)]$, we obtain $K \cap W(M, N) \in \mathcal{F} \backslash\{\emptyset\}$; then, $S_{o}(K \cap W(M, N), \kappa) \in$ $\mathcal{F} \backslash\{\emptyset\}$ for $\kappa \in] 0, \infty[$.

Theorem 2. If $\mathbf{K} \in \mathcal{K}[W(M, N)]$, then $\forall \varepsilon \in] 0, \infty[\exists \delta \in] 0, \infty[\forall \widetilde{M} \in \mathcal{F} \forall \widetilde{N} \in \mathcal{F}$

$$
\begin{gathered}
\left(\left(M \subset \widetilde{M} \subset S_{o}(M, \delta)\right) \&\left(N \subset \widetilde{N} \subset S_{o}(N, \delta)\right)\right) \Longrightarrow \\
\Longrightarrow\left(\mathbf{K} \cap W(M, N) \subset \mathbf{K} \cap W(\widetilde{M}, \widetilde{N}) \subset S_{o}(\mathbf{K} \cap W(M, N), \varepsilon)\right) .
\end{gathered}
$$

Proof. Fix $\mathbf{K} \in \mathcal{K}[W(M, N)]$. Then, for all $\widetilde{M} \in \mathcal{F}$ and $\widetilde{N} \in \mathcal{F}$,

$$
((M \subset \widetilde{M}) \&((N \subset \widetilde{N})) \Longrightarrow(\mathbf{K} \cap W(M, N) \subset \mathbf{K} \cap W(\widetilde{M}, \widetilde{N}))
$$

Fix $\varepsilon \in] 0, \infty[$. We show that $\exists \delta \in] 0, \infty[\forall \widetilde{M} \in \mathcal{F} \forall \widetilde{N} \in \mathcal{F}$

$$
\left(\left(\widetilde{M} \subset S_{o}(M, \delta)\right) \&\left(\widetilde{N} \subset S_{o}(N, \delta)\right)\right) \Longrightarrow\left(\mathbf{K} \cap W(\widetilde{M}, \widetilde{N}) \subset S_{o}(\mathbf{K} \cap W(M, N), \varepsilon)\right)
$$

Let us suppose the contrary: $\forall \delta \in] 0, \infty[\exists \widetilde{M} \in \mathcal{F} \exists \widetilde{N} \in \mathcal{F}$

$$
\left(\left(\widetilde{M} \subset S_{o}(M, \delta)\right) \&\left(\widetilde{N} \subset S_{o}(N, \delta)\right) \&\left((\mathbf{K} \cap W(\widetilde{M}, \widetilde{N})) \backslash S_{o}(\mathbf{K} \cap W(M, N), \varepsilon) \neq \emptyset\right)\right.
$$

As a corollary (see (5.8)), by (7.5), we find that $\forall \delta \in] 0, \infty[$

$$
\left(\mathbf{K} \cap W\left(S_{o}(M, \delta), S_{o}(N, \delta)\right)\right) \backslash S_{o}(\mathbf{K} \cap W(M, N), \varepsilon) \neq \emptyset .
$$

Thus, in particular, by the axiom of choice, the following property holds:

$$
\prod_{k \in \mathbb{N}}\left(\left(\mathbf{K} \cap W\left(S_{o}\left(M, \frac{1}{k}\right), S_{o}\left(N, \frac{1}{k}\right)\right)\right) \backslash S_{o}(\mathbf{K} \cap W(M, N), \varepsilon)\right) \neq \emptyset .
$$

Using (7.6), we choose a sequence

$$
\left(\left(t^{(k)}, x^{(k)}\right)\right)_{k \in \mathbb{N}} \in \prod_{k \in \mathbb{N}}\left(\left(\mathbf{K} \cap W\left(S_{o}\left(M, \frac{1}{k}\right), S_{o}\left(N, \frac{1}{k}\right)\right)\right) \backslash S_{o}(\mathbf{K} \cap W(M, N), \varepsilon)\right) .
$$

It is easy to see that $k \mapsto\left(t^{(k)}, x^{(k)}\right): \mathbb{N} \rightarrow \mathbf{K}$. In addition,

$$
\left(t^{(j)}, x^{(j)}\right) \in W\left(S_{o}\left(M, \frac{1}{j}\right), S_{o}\left(N, \frac{1}{j}\right)\right) \backslash S_{o}(\mathbf{K} \cap W(M, N), \varepsilon) \quad \forall j \in \mathbb{N} .
$$

Since $\mathbf{K} \in(\mathbf{t}-$ comp $)\left[T \times \mathbb{R}^{n}\right]$, for a sequence $\left(\zeta_{i}\right)_{i \in \mathbb{N}} \in \mathbb{N}^{\mathbb{N}}$ with the property

$$
\zeta_{j}<\zeta_{j+1} \quad \forall j \in \mathbb{N}
$$


and a position $\left(t_{*}, x_{*}\right) \in \mathbf{K}$, the convergence

$$
\left(\rho\left(\left(t^{\left(\zeta_{s}\right)}, x^{\left(\zeta_{s}\right)}\right),\left(t_{*}, x_{*}\right)\right)\right)_{s \in \mathbb{N}} \longrightarrow 0
$$

holds. Then, for $k \in \mathbb{N}$, we find that $k \leqslant \zeta_{k}$ and

$$
\left(S_{o}\left(M, \frac{1}{\zeta_{k}}\right) \subset S_{o}\left(M, \frac{1}{k}\right)\right) \&\left(S_{o}\left(N, \frac{1}{\zeta_{k}}\right) \subset S_{o}\left(N, \frac{1}{k}\right)\right) .
$$

Therefore, by (7.8), the inclusions

$$
\left(t^{\left(\zeta_{s}\right)}, x^{\left(\zeta_{s}\right)}\right) \in W\left(S_{o}\left(M, \frac{1}{s}\right), S_{o}\left(N, \frac{1}{s}\right)\right) \backslash S_{o}(\mathbf{K} \cap W(M, N), \varepsilon) \quad \forall s \in \mathbb{N}
$$

hold. Using (5.8), from (7.10), we find that, for all $r \in \mathbb{N}$,

$$
\left(t^{\left(\zeta_{s}\right)}, x^{\left(\zeta_{s}\right)}\right) \in W\left(S_{o}\left(M, \frac{1}{r}\right), S_{o}\left(N, \frac{1}{r}\right)\right) \quad \forall s \in \overrightarrow{r, \infty},
$$

where $W\left(S_{o}\left(M, \frac{1}{r}\right), S_{o}\left(N, \frac{1}{r}\right)\right) \in \mathcal{F}$ (see (6.6), (7.2)). From (7.9) and (7.11), the inclusion $\left(t_{*}, x_{*}\right) \in$ $W\left(S_{o}\left(M, \frac{1}{r}\right), S_{o}\left(N, \frac{1}{r}\right)\right)$ follows. Since the choice of $r$ was arbitrary, we establish that

$$
\left(t_{*}, x_{*}\right) \in W\left(S_{o}\left(M, \frac{1}{k}\right), S_{o}\left(N, \frac{1}{k}\right)\right) \quad \forall k \in \mathbb{N} .
$$

We note that (since $M \in \mathcal{F}$ and $N \in \mathcal{F}$ )

$$
\left(\left(S_{o}\left(M, \frac{1}{k}\right)\right)_{k \in \mathbb{N}} \downarrow M\right) \&\left(\left(S_{o}\left(N, \frac{1}{k}\right)\right)_{k \in \mathbb{N}} \downarrow N\right)
$$

By Theorem 1 and (7.2), we obtain

$$
\left(W\left(S_{o}\left(M, \frac{1}{k}\right), S_{o}\left(N, \frac{1}{k}\right)\right)\right)_{k \in \mathbb{N}} \downarrow W(M, N) .
$$

As a corollary, the following equality is realized:

$$
W(M, N)=\bigcap_{k \in \mathbb{N}} W\left(S_{o}\left(M, \frac{1}{k}\right), S_{o}\left(N, \frac{1}{k}\right)\right) .
$$

From (7.12) and (7.13), the inclusion $\left(t_{*}, x_{*}\right) \in W(M, N)$ follows. Therefore,

$$
\left(t_{*}, x_{*}\right) \in \mathbf{K} \cap W(M, N) .
$$

We recall that, by (7.10),

$$
\left(t^{\left(\zeta_{s}\right)}, x^{\left(\zeta_{s}\right)}\right) \notin S_{o}(\mathbf{K} \cap W(M, N), \varepsilon) \quad \forall s \in \mathbb{N} .
$$

From (7.2) and (7.15), we obtain the following inequalities:

$$
\varepsilon<\rho\left(\left(t^{\left(\zeta_{s}\right)}, x^{\left(\zeta_{s}\right)}\right) ; \mathbf{K} \cap W(M, N)\right) \quad \forall s \in \mathbb{N} .
$$

In addition, in the form of

$$
(t, x) \longmapsto \rho((t, x) ; \mathbf{K} \cap W(M, N)): T \times \mathbb{R}^{n} \longrightarrow[0, \infty[,
$$

we have a continuous function. Therefore, from (7.9) and (7.16), we obtain

$$
\varepsilon \leqslant \rho\left(\left(t_{*}, x_{*}\right) ; \mathbf{K} \cap W(M, N)\right),
$$


where $\varepsilon>0$. By (7.17), the property

$$
\left(t_{*}, x_{*}\right) \notin \mathbf{K} \cap W(M, N)
$$

holds. We obtain the obvious contradiction with (7.14). This contradiction proves (7.4). Now, we use (7.3).

We have obtained the distinctive "quasicontinuous from above" property of the mapping

$$
(\widetilde{M}, \widetilde{N}) \longmapsto W(\widetilde{M}, \widetilde{N}): \mathcal{F} \times \mathcal{F} \longrightarrow \mathcal{F}
$$

at the point $(M, N)$ for which $W(M, N) \neq \emptyset$ (in this case, $\mathcal{K}[W(M, N)] \neq \emptyset$ ).

If is useful to note the following property. If $\mathbf{K} \in \mathcal{K}[W(M, N)]$, then, for $\widetilde{M} \in \mathcal{F}$ and $\widetilde{N} \in \mathcal{F}$ such that

$$
(M \subset \widetilde{M}) \&(N \subset \widetilde{N})
$$

$\mathbf{K} \cap W(\widetilde{M}, \widetilde{N}) \in(\mathbf{t}-\mathrm{comp})\left[T \times \mathbb{R}^{n}\right]$. Therefore, Theorem 2 can be considered a statement about "quasicontinuity from above" at the point $(M, N)$ in the sense of a Hausdorff metric. We recall that, in [17, Section 8], an example was presented, which showed that mapping (7.18) may not have the ordinary (local) continuity at the fixed above-mentioned point $(M, N)$.

In conclusion of this section, we note one property established during the proof of Theorem 2. Namely, for $M \in \mathcal{F}$ and $N \in \mathcal{F}$ with the property $W(M, N) \neq \emptyset$, we have

$$
\begin{gathered}
\forall \mathbf{K} \in \mathcal{K}[W(M, N)] \forall \varepsilon \in] 0, \infty[\exists \delta \in] 0, \infty[\forall \widetilde{M} \in \mathcal{F} \forall \widetilde{N} \in \mathcal{F} \\
\left(\left(\widetilde{M} \subset S_{o}(M, \delta)\right) \&\left(\widetilde{N} \subset S_{o}(N, \delta)\right)\right) \Longrightarrow\left(\mathbf{K} \cap W(\widetilde{M}, \widetilde{N}) \subset S_{o}(\mathbf{K} \cap W(M, N), \varepsilon)\right) .
\end{gathered}
$$

\section{Topological properties of fragments of the set of positional absorption, 2}

Here we consider an analog of Theorem 2 for sections of the set of positional absorption in a more general case. For this we introduce a new notation.

If $H \in \mathcal{P}^{\prime}\left(\mathbb{R}^{n}\right)$ and $x \in \mathbb{R}^{n}$, define

$$
(\|\cdot\|-\inf )[x ; H] \triangleq \inf (\{\|x-h\|: h \in H\}) ;
$$

of course, (8.1) is a nonnegative number. We note that, for all $H \in \mathcal{P}^{\prime}\left(\mathbb{R}^{n}\right)$,

$$
x \longmapsto(\|\cdot\|-\inf )[x ; H]: \mathbb{R}^{n} \longrightarrow[0, \infty[
$$

is a continuous function. Therefore,

$$
B_{n}^{o}(L, \varepsilon) \triangleq\left\{x \in \mathbb{R}^{n} \mid(\|\cdot\|-\inf )[x ; L] \leqslant \varepsilon\right\} \in \mathbf{F} \backslash\{\emptyset\} \quad \forall L \in \mathcal{P}^{\prime}\left(\mathbb{R}^{n}\right) \quad \forall \varepsilon \in[0, \infty[
$$

(in (8.3), we consider continuous inverse images of closed semi-infinite intervals of real line).

If $\Lambda \in \mathcal{P}\left(T \times \mathbb{R}^{n}\right)$, define

$$
\operatorname{Supp}(\Lambda) \triangleq\{t \in T \mid \Lambda\langle t\rangle \neq \emptyset\} .
$$

Consequently, for $\Lambda \in \mathcal{P}\left(T \times \mathbb{R}^{n}\right)$ and $t \in \operatorname{Supp}(\Lambda)$, we obtain $\Lambda\langle t\rangle \in \mathcal{P}^{\prime}\left(\mathbb{R}^{n}\right)$ and, for $\varepsilon \in[0, \infty[$, by (8.3), the set $B_{n}^{o}(\Lambda\langle t\rangle, \varepsilon) \in \mathbf{F} \backslash\{\emptyset\}$ is well-defined. Then,

$$
\mathbb{S}(H, \varepsilon) \triangleq\left\{(t, x) \in \operatorname{Supp}(H) \times \mathbb{R}^{n} \mid x \in B_{n}^{o}(H\langle t\rangle, \varepsilon)\right\} \in \mathcal{P}\left(T \times \mathbb{R}^{n}\right) \quad \forall H \in \mathcal{P}\left(T \times \mathbb{R}^{n}\right) \quad \forall \varepsilon \in[0, \infty[.
$$

From (8.4), we obtain $\left.\forall H \in \mathcal{P}\left(T \times \mathbb{R}^{n}\right) \quad \forall \varepsilon \in\right] 0, \infty[$

$$
\left(\mathbb{S}(H, \varepsilon)\langle t\rangle=B_{n}^{o}(H\langle t\rangle, \varepsilon) \quad \forall t \in \operatorname{Supp}(H)\right) \&(\mathbb{S}(H, \varepsilon)\langle\tilde{t}\rangle=\emptyset \quad \forall \tilde{t} \in T \backslash \operatorname{Supp}(H)) .
$$


Certainly, $\mathbb{S}(H, \varepsilon)\langle t\rangle \in \mathbf{F} \quad \forall H \in \mathcal{P}\left(T \times \mathbb{R}^{n}\right) \quad \forall \varepsilon \in[0, \infty[\forall t \in T$. From statements of Section 4, we obtain

$$
\mathbb{S}(H, \varepsilon) \in \mathfrak{F} \quad \forall H \in \mathcal{P}\left(T \times \mathbb{R}^{n}\right) \quad \forall \varepsilon \in[0, \infty[.
$$

Assume that, for $F \in \mathbf{F} \backslash\{\emptyset\}$,

$$
\mathfrak{K}[F] \triangleq\left\{K \in\left(\tau_{\mathbb{R}}^{(n)}-\operatorname{comp}\right)\left[\mathbb{R}^{n}\right] \mid K \cap F \neq \emptyset\right\} .
$$

We fix $M \in \mathcal{F} \backslash\{\emptyset\}$ and $N \in \mathfrak{F}$ until the end of this section. In addition, for $t \in \operatorname{Supp}(W(M, N))$, we obtain $W(M, N)\langle t\rangle \in \mathbf{F} \backslash\{\emptyset\}$ (see (5.9)) and, as a corollary,

$$
\mathfrak{K}[W(M, N)\langle t\rangle] \in \mathcal{P}^{\prime}\left(\left(\tau_{\mathbb{R}}^{(n)}-\operatorname{comp}\right)\left[\mathbb{R}^{n}\right]\right) ;
$$

therefore, $K \cap W(M, N)\langle t\rangle \in \mathbf{F} \backslash\{\emptyset\}$ for $K \in \mathfrak{K}[W(M, N)\langle t\rangle]$.

Proposition 6. Let $t_{*} \in \operatorname{Supp}(W(M, N))$ and $\mathbb{K} \in \mathfrak{K}\left[W(M, N)\left\langle t_{*}\right\rangle\right]$. Then, $\left.\forall \varepsilon \in\right] 0, \infty[\exists \delta \in$ ] $0, \infty[\forall \widetilde{M} \in \mathcal{F} \forall \widetilde{N} \in \mathfrak{F}$

$$
\begin{gathered}
\left(\left(M \subset \widetilde{M} \subset S_{o}(M, \delta)\right) \&(N \subset \widetilde{N} \subset \mathbb{S}(N, \delta))\right) \Longrightarrow \\
\Longrightarrow\left(\mathbb{K} \cap W(M, N)\left\langle t_{*}\right\rangle \subset \mathbb{K} \cap W(\widetilde{M}, \widetilde{N})\left\langle t_{*}\right\rangle \subset B_{n}^{o}\left(\mathbb{K} \cap W(M, N)\left\langle t_{*}\right\rangle, \varepsilon\right)\right) .
\end{gathered}
$$

Proof. Fix $\varepsilon \in] 0, \infty[$. Then, by (8.3), we have

$$
B_{n}^{o}\left(\mathbb{K} \cap W(M, N)\left\langle t_{*}\right\rangle, \varepsilon\right)=\left\{x \in \mathbb{R}^{n} \mid(\|\cdot\|-\inf )\left[x ; \mathbb{K} \cap W(M, N)\left\langle t_{*}\right\rangle\right] \leqslant \varepsilon\right\} \in \mathbf{F} \backslash\{\emptyset\} .
$$

We show that $\exists \delta \in] 0, \infty[\forall \widetilde{M} \in \mathcal{F} \forall \widetilde{N} \in \mathfrak{F}$

$$
\left(\left(\widetilde{M} \subset S_{o}(M, \delta)\right) \&(\widetilde{N} \subset \mathbb{S}(N, \delta))\right) \Longrightarrow\left(\mathbb{K} \cap W(\widetilde{M}, \widetilde{N})\left\langle t_{*}\right\rangle \subset B_{n}^{o}\left(\mathbb{K} \cap W(M, N)\left\langle t_{*}\right\rangle, \varepsilon\right)\right)
$$

Let us assume the contrary: $\forall \delta \in] 0, \infty[\exists \widetilde{M} \in \mathcal{F} \exists \widetilde{N} \in \mathfrak{F}$ :

$$
\left(\widetilde{M} \subset S_{o}(M, \delta)\right) \&(\widetilde{N} \subset \mathbb{S}(N, \delta)) \&\left(\left(\mathbb{K} \cap W(\widetilde{M}, \widetilde{N})\left\langle t_{*}\right\rangle\right) \backslash B_{n}^{o}\left(\mathbb{K} \cap W(M, N)\left\langle t_{*}\right\rangle, \varepsilon\right) \neq \emptyset\right)
$$

Then, by (4.2), (5.8), and (8.10), we obtain $\forall \delta \in] 0, \infty[$

$$
\left(\mathbb{K} \cap W\left(S_{o}(M, \delta), \mathbb{S}(N, \delta)\right)\left\langle t_{*}\right\rangle\right) \backslash B_{n}^{o}\left(\mathbb{K} \cap W(M, N)\left\langle t_{*}\right\rangle, \varepsilon\right) \neq \emptyset .
$$

As a corollary (we use the axiom of choice),

$$
\prod_{k \in \mathbb{N}}\left(\left(\mathbb{K} \cap W\left(S_{o}\left(M, \frac{1}{k}\right), \mathbb{S}\left(N, \frac{1}{k}\right)\right)\left\langle t_{*}\right\rangle\right) \backslash B_{n}^{o}\left(\mathbb{K} \cap W(M, N)\left\langle t_{*}\right\rangle, \varepsilon\right)\right) \neq \emptyset .
$$

Using (8.12), we choose an arbitrary sequence such that

$$
\left(x^{(k)}\right)_{k \in \mathbb{N}} \in \prod_{k \in \mathbb{N}}\left(\left(\mathbb{K} \cap W\left(S_{o}\left(M, \frac{1}{k}\right), \mathbb{S}\left(N, \frac{1}{k}\right)\right)\left\langle t_{*}\right\rangle\right) \backslash B_{n}^{o}\left(\mathbb{K} \cap W(M, N)\left\langle t_{*}\right\rangle, \varepsilon\right)\right) .
$$

From (8.13), we find that (in particular)

$$
\left(x^{(k)}\right)_{k \in \mathbb{N}}: \mathbb{N} \longrightarrow \mathbb{K} .
$$

In addition, the following inclusions hold:

$$
x^{(s)} \in\left(\mathbb{K} \cap W\left(S_{o}\left(M, \frac{1}{s}\right), \mathbb{S}\left(N, \frac{1}{s}\right)\right)\left\langle t_{*}\right\rangle\right) \backslash B_{n}^{o}\left(\mathbb{K} \cap W(M, N)\left\langle t_{*}\right\rangle, \varepsilon\right) \quad \forall s \in \mathbb{N} .
$$


We recall that $\mathbb{K} \in\left(\tau_{\mathbb{R}}^{(n)}-\right.$ comp $)\left[\mathbb{R}^{n}\right]$. Therefore, we can choose a sequence $\left(\omega_{k}\right)_{k \in \mathbb{N}} \in \mathbb{N}^{\mathbb{N}}$ and a point $x_{*} \in \mathbb{K}$ such that

$$
\left(\omega_{s}<\omega_{s+1} \quad \forall s \in \mathbb{N}\right) \&\left(\left(\left\|x^{\left(\omega_{s}\right)}-x_{*}\right\|\right)_{s \in \mathbb{N}} \longrightarrow 0\right) .
$$

We note that $k \leqslant \omega_{k} \forall k \in \mathbb{N}$. Therefore (see (5.8)), we obtain

$$
x^{\left(\omega_{k}\right)} \in\left(\mathbb{K} \cap W\left(S_{o}\left(M, \frac{1}{k}\right), \mathbb{S}\left(N, \frac{1}{k}\right)\right)\left\langle t_{*}\right\rangle\right) \backslash B_{n}^{o}\left(\mathbb{K} \cap W(M, N)\left\langle t_{*}\right\rangle, \varepsilon\right) \quad \forall k \in \mathbb{N} .
$$

Let $r \in \mathbb{N}$. Then, from (5.8) and (8.17), we obtain

$$
x^{\left(\omega_{k}\right)} \in W\left(S_{o}\left(M, \frac{1}{r}\right), \mathbb{S}\left(N, \frac{1}{r}\right)\right)\left\langle t_{*}\right\rangle \forall k \in \overrightarrow{r, \infty} .
$$

In addition, by (5.11) and (7.2),

$$
W\left(S_{o}\left(M, \frac{1}{r}\right), \mathbb{S}\left(N, \frac{1}{r}\right)\right)\left\langle t_{*}\right\rangle \in \mathbf{F} .
$$

Therefore, from (8.16) and (8.18), the inclusion

$$
x_{*} \in W\left(S_{o}\left(M, \frac{1}{r}\right), \mathbb{S}\left(N, \frac{1}{r}\right)\right)\left\langle t_{*}\right\rangle
$$

follows. As a corollary, $\left(t_{*}, x_{*}\right) \in W\left(S_{o}\left(M, \frac{1}{r}\right), \mathbb{S}\left(N, \frac{1}{r}\right)\right)$. Since the choice of $r$ was arbitrary, we obtain

$$
\left(t_{*}, x_{*}\right) \in W\left(S_{o}\left(M, \frac{1}{k}\right), \mathbb{S}\left(N, \frac{1}{k}\right)\right) \quad \forall k \in \mathbb{N} .
$$

We note that $\left(S_{o}\left(M, \frac{1}{k}\right)\right)_{k \in \mathbb{N}} \downarrow M$. Moreover, it is easy to establish (see (8.3) and (8.4)) that

$$
\left(\mathbb{S}\left(N, \frac{1}{k}\right)\right)_{k \in \mathbb{N}} \downarrow N
$$

Therefore, by Theorem 1, we obtain

$$
\left(W\left(S_{o}\left(M, \frac{1}{k}\right), \mathbb{S}\left(N, \frac{1}{k}\right)\right)\right)_{k \in \mathbb{N}} \downarrow W(M, N) .
$$

In particular, the following equality is realized:

$$
W(M, N)=\bigcap_{k \in \mathbb{N}} W\left(S_{o}\left(M, \frac{1}{k}\right), \mathbb{S}\left(N, \frac{1}{k}\right)\right) .
$$

From (8.19) and (8.20), we see that $\left(t_{*}, x_{*}\right) \in W(M, N)$; consequently,

$$
x_{*} \in \mathbb{K} \cap W(M, N)\left\langle t_{*}\right\rangle .
$$

On the other hand, by (8.17), the following properties hold:

$$
x^{\left(\omega_{s}\right)} \in \mathbb{K} \backslash \mathbb{B}_{n}^{o}\left(\mathbb{K} \cap W(M, N)\left\langle t_{*}\right\rangle, \varepsilon\right) \quad \forall s \in \mathbb{N} .
$$

Since $\mathbb{K} \subset \mathbb{R}^{n}$, from (8.3) and (8.22), we have the following system of inequalities:

$$
\varepsilon<(\|\cdot\|-\inf )\left[x^{\left(\omega_{s}\right)} ; \mathbb{K} \cap W(M, N)\left\langle t_{*}\right\rangle\right] \forall s \in \mathbb{N} .
$$

Since functions of type (8.2) are continuous, (8.16) and (8.23) imply that

$$
\varepsilon \leqslant(\|\cdot\|-\inf )\left[x_{*} ; \mathbb{K} \cap W(M, N)\left\langle t_{*}\right\rangle\right]
$$


But, from (8.24), the property $x_{*} \notin \mathbb{K} \cap W(M, N)\left\langle t_{*}\right\rangle$ follows in spite of (8.21). The obtained contradiction proves (8.9). Later, using (5.8), we obtain (8.7).

We note the following important corollary of Proposition 6: if $t_{o} \in \operatorname{Supp}(W(M, N))$ and $\mathbb{K} \in$ $\mathfrak{K}\left[W(M, N)\left\langle t_{o}\right\rangle\right]$, then $\left.\forall \varepsilon \in\right] 0, \infty[\exists \delta \in] 0, \infty[\forall \widetilde{M} \in \mathcal{F} \forall \widetilde{N} \in \mathfrak{F}$

$$
\begin{gathered}
\left(\left(M \subset \widetilde{M} \subset S_{o}(M, \delta)\right) \&((N \subset \widetilde{N} \subset \mathbb{S}(N, \delta))) \Longrightarrow\right. \\
\Longrightarrow\left(\mathbb{K} \cap W(M, N)\left\langle t_{o}\right\rangle \subset \mathbb{K} \cap W(\widetilde{M}, \widetilde{N})\left\langle t_{o}\right\rangle \subset B_{n}^{o}\left(\mathbb{K} \cap W(M, N)\left\langle t_{o}\right\rangle, \varepsilon\right)\right) .
\end{gathered}
$$

In fact, in (8.25), we view $t_{o}$ as an actual initial time and estimate if it is possible to approximate the set $W(M, N)\left\langle t_{o}\right\rangle$ from above. We recall that, during the proof of Proposition 6 (in fact), the following property is established: let $t_{o} \in \operatorname{Supp}(W(M, N))$ and $\mathbb{K} \in \mathfrak{K}\left[W(M, N)\left\langle t_{o}\right\rangle\right]$; then, $\forall \varepsilon \in$ ] $0, \infty[\exists \delta \in] 0, \infty[\forall \widetilde{M} \in \mathcal{F} \forall \widetilde{N} \in \mathfrak{F}$

$$
\left(\left(\widetilde{M} \subset S_{o}(M, \delta)\right) \&((\widetilde{N} \subset \mathbb{S}(N, \delta))) \Longrightarrow\left(\mathbb{K} \cap W(\widetilde{M}, \widetilde{N})\left\langle t_{o}\right\rangle \subset B_{n}^{o}\left(\mathbb{K} \cap W(M, N)\left\langle t_{o}\right\rangle, \varepsilon\right)\right)\right.
$$

\section{Generalized set-valued quasistrategies and the set of positional absorption}

In this section, we investigate the means of successfully solving the guidance problem in the class of quasistrategies. These quasistrategies are defined as multifunctions operating in measure spaces. We use definitions of Section 3. If $t_{*} \in T$, then

$$
\begin{gathered}
\widetilde{A}_{t_{*}} \triangleq\left\{\alpha \in \prod_{\nu \in \mathcal{E}_{t_{*}}} \mathcal{P}^{\prime}\left(\Pi_{t_{*}}(\nu)\right)\left|\forall \nu_{1} \in \mathcal{E}_{t_{*}} \forall \nu_{2} \in \mathcal{E}_{t_{*}} \forall \theta \in\left[t_{*}, \vartheta_{o}\right]\right|\right. \\
\left.\left(\left(\nu_{1} \mid \mathcal{D}_{t_{*}}^{(\theta)}\right)=\left(\nu_{2} \mid \mathcal{D}_{t_{*}}^{(\theta)}\right)\right) \Longrightarrow\left(\left[\alpha\left(\nu_{1}\right) \mid t_{*}, \theta\right)=\left[\alpha\left(\nu_{2}\right) \mid t_{*}, \theta\right)\right)\right\} .
\end{gathered}
$$

In (9.1), we introduce the set of all (generalized set-valued) quasistrategies of player I on a (closed) interval $\left[t_{*}, \vartheta_{o}\right]$. Thus, if $t_{*} \in T$ and $\alpha \in \widetilde{A}_{t_{*}}$, then

$$
\alpha: \mathcal{E}_{t_{*}} \longrightarrow \mathcal{P}^{\prime}\left(\mathcal{H}_{t_{*}}\right)
$$

for which $\alpha(\nu) \subset \Pi_{t_{*}}(\nu) \forall \nu \in \mathcal{E}_{t_{*}}$. From definitions of Section 3, we obtain (see [9])

$$
\Pi_{t_{*}}(\cdot) \triangleq\left(\Pi_{t_{*}}(\nu)\right)_{\nu \in \mathcal{E}_{t_{*}}} \in \widetilde{A}_{t_{*}} \forall t_{*} \in T
$$

So, $\widetilde{A}_{t} \neq \emptyset \quad \forall t \in T$. We obtain

$$
\widetilde{\Pi}_{t}(\alpha) \triangleq \bigcup_{\nu \in \mathcal{E}_{t}} \alpha(\nu) \in \mathcal{P}^{\prime}\left(\mathcal{H}_{t}\right) \quad \forall t \in T \quad \forall \alpha \in \widetilde{A}_{t}
$$

In (9.2), the set of all possible generalized controls for the choice of a fixed quasistrategy is introduced.

Using properties noted in Section 3, we obtain, for $t \in T$, the following fact:

$$
(\mathcal{H}-\operatorname{clos})_{*}[t] \triangleq\left\{H \in \mathcal{P}\left(\mathcal{H}_{t}\right) \mid \forall\left(\eta_{k}\right)_{k \in \mathbb{N}} \in H^{\mathbb{N}} \forall \eta \in \mathcal{H}_{t} \quad\left(\left(\eta_{k}\right)_{k \in \mathbb{N}} \rightarrow \eta\right) \Longrightarrow(\eta \in H)\right\}
$$

is the family of all *-weak closed subsets of $\mathcal{H}_{t}$. We suppose that

$$
\widetilde{A}_{t}^{\Pi} \triangleq\left\{\alpha \in \widetilde{A}_{t} \mid \widetilde{\Pi}_{t}(\alpha) \in(\mathcal{H}-\operatorname{clos})_{*}[t]\right\} \quad \forall t \in T .
$$

We call elements of sets (9.4) quasi-programs on the corresponding time interval. From (9.2), we obtain $\Pi_{t}(\cdot) \in \widetilde{A}_{t}^{\Pi} \forall t \in T$. So, $\widetilde{A}_{t}^{\Pi} \neq \emptyset \forall t \in T$. We recall the following property of [17, Section 10]: if $t \in T, \alpha \in \widetilde{A}_{t}, \nu \in \mathcal{E}_{t}, \eta \in \alpha(\nu)$, and $\theta \in\left[t, \vartheta_{o}\right]$, then

$$
\left(\left\{\tilde{\eta} \in \Pi_{\theta}(\tilde{\nu}) \mid \eta \perp \tilde{\eta} \in \alpha(\nu \bowtie \tilde{\nu})\right\}\right)_{\tilde{\nu} \in \mathcal{E}_{\theta}} \in \widetilde{A}_{\theta} .
$$


Evidently, (9.5) can be viewed as a restriction of the quasistrategy $\alpha$ onto the interval $\left[\theta, \vartheta_{o}\right]$.

In the following, we fix $M \in \mathcal{F}$ and $N \in \mathfrak{F}$. We assume that

$$
\begin{gathered}
\mathfrak{H}_{M, N}(t, x) \triangleq\left\{\eta \in \mathcal{H}_{t} \mid \forall \vartheta \in\left[t, \vartheta_{o}\right] \quad((\vartheta, \varphi(\vartheta, t, x, \eta)) \in M) \Longrightarrow\right. \\
\Longrightarrow(\exists \xi \in[t, \vartheta[:(\xi, \varphi(\xi, t, x, \eta)) \notin N)\} \forall(t, x) \in N .
\end{gathered}
$$

Using (9.5), in [17, Section 10], we established the following property:

$$
\widetilde{\Pi}_{t}(\alpha) \cap \mathfrak{H}_{M, N}(t, x) \neq \emptyset \quad \forall k \in \mathbb{N}_{o} \quad \forall(t, x) \in N \backslash W_{k}(M, N) \quad \forall \alpha \in \widetilde{A}_{t} .
$$

Using (5.5) and (9.7), we obtain

$$
\widetilde{\Pi}_{t}(\alpha) \cap \mathfrak{H}_{M, N}(t, x) \neq \emptyset \quad \forall(t, x) \in N \backslash W(M, N) \quad \forall \alpha \in \widetilde{A}_{t} .
$$

Thus, for every position of $N \backslash W(M, N)$, the guidance problem in the class of quasistrategies is unsolvable. Now, we introduce the set

$$
\begin{gathered}
\mathcal{S}_{M, N}(t, x) \triangleq\left\{\eta \in \mathcal{H}_{t} \mid \exists \theta \in\left[t, \vartheta_{o}\right]:((\theta, \varphi(\theta, t, x, \eta)) \in M) \&\right. \\
\&((\xi, \varphi(\xi, t, x, \eta)) \in N \quad \forall \xi \in[t, \theta[)\} \quad \forall(t, x) \in N .
\end{gathered}
$$

That is, in (9.9), we introduce the sets, all elements of which are generalized controls with the guidance property for fixed $M$ and $N$. In connection with (9.9), we assume that

$$
\begin{aligned}
& \pi_{t, x}^{(W)}\langle\nu \mid M, N\rangle \triangleq\left\{\eta \in \Pi_{t}(\nu) \mid \exists \vartheta \in\left[t, \vartheta_{o}\right]:((\vartheta, \varphi(\vartheta, t, x, \eta)) \in M) \&\right. \\
& \&(\xi, \varphi(\xi, t, x, \eta)) \in W(M, N) \quad \forall \xi \in[t, \vartheta[)\} \quad \forall(t, x) \in N \quad \forall \nu \in \mathcal{E}_{t} .
\end{aligned}
$$

In terms of (9.10), we construct special multifunctions. Namely, for $(t, x) \in N$, we obtain

$$
\pi_{t, x}^{(W)}\langle\cdot \mid M, N\rangle \triangleq\left(\pi_{t, x}^{(W)}\langle\nu \mid M, N\rangle\right)_{\nu \in \mathcal{E}_{t}} \in \prod_{\nu \in \mathcal{E}_{t}} \mathcal{P}\left(\Pi_{t}(\nu)\right) .
$$

It is easily established (see [17, section 10$]$ ) that

$$
\pi_{t, x}^{(W)}\langle\cdot \mid M, N\rangle \in \widetilde{A}_{t}^{\Pi} \forall(t, x) \in W(M, N) .
$$

From (9.3), (9.4), and (9.11), we find that, for $(t, x) \in W(M, N)$, the set

$$
\widetilde{\Pi}_{t}\left(\pi_{t, x}^{(W)}\langle\cdot \mid M, N\rangle\right) \in(\mathcal{H}-\operatorname{clos})_{*}[t]
$$

is well-defined. Moreover, in [17, Corollary 10.2], the following important property was obtained:

$$
\widetilde{\Pi}_{t_{*}}\left(\pi_{t_{*}, x_{*}}^{(W)}\langle\cdot \mid M, N\rangle\right) \subset \mathcal{S}_{M, N}\left(t_{*}, x_{*}\right) \quad \forall\left(t_{*}, x_{*}\right) \in W(M, N) .
$$

Theorem 3. The set $W(M, N)$ exhausts the possibilities of player I in solution of the guidance problem in the classes of quasistrategies and quasi-programs:

$$
\begin{gathered}
W(M, N)=\left\{(t, x) \in N \mid \exists \alpha \in \widetilde{A}_{t}: \widetilde{\Pi}_{t}(\alpha) \subset \mathcal{S}_{M, N}(t, x)\right\}= \\
=\left\{(t, x) \in N \mid \exists \alpha \in \widetilde{A}_{t}^{\Pi}: \widetilde{\Pi}_{t}(\alpha) \subset \mathcal{S}_{M, N}(t, x)\right\}
\end{gathered}
$$


This theorem is established in [17, Section 10] and (in fact) in [9]; (9.12) defines a concrete variant of quasi-program that solves the problem. We note that this variant has the property of extremality in the ordinal sense. In this connection, we assume that, for all $t \in T$, the binary relation $\stackrel{t}{\sqsubseteq}$ on the set $\widetilde{A}_{t}$ is defined by the following rule: $\forall \alpha_{1} \in \widetilde{A}_{t} \forall \alpha_{2} \in \widetilde{A}_{t}$

$$
\left(\alpha_{1} \stackrel{t}{\sqsubseteq} \alpha_{2}\right) \stackrel{\text { def }}{\Longleftrightarrow}\left(\alpha_{1}(\nu) \subset \alpha_{2}(\nu) \quad \forall \nu \in \mathcal{E}_{t}\right) ;
$$

clearly, (9.13) defines a partial order on $\widetilde{A}_{t}$.

Theorem 4. If $\left(t_{*} x_{*}\right) \in W(M, N)$ and $\alpha \in \widetilde{A}_{t_{*}}$, then

$$
\left(\widetilde{\Pi}_{t_{*}}(\alpha) \subset \mathcal{S}_{M, N}\left(t_{*}, x_{*}\right)\right) \Longrightarrow\left(\alpha \sqsubseteq \pi_{t_{*}, x_{*}}^{(W)}\langle\cdot \mid M, N\rangle\right) .
$$

Proof. Fix $\left(t_{*}, x_{*}\right) \in W(M, N)$ and $\alpha \in \widetilde{A}_{t_{*}}$ such that

$$
\widetilde{\Pi}_{t_{*}}(\alpha) \subset \mathcal{S}_{M, N}\left(t_{*}, x_{*}\right) .
$$

We show that $\alpha \stackrel{t_{*}}{\sqsubseteq} \pi_{t_{*}, x_{*}}^{(W)}\langle\cdot \mid M, N\rangle$. Indeed, let us assume the contrary. Then, by (9.13),

$$
\exists \nu \in \mathcal{E}_{t_{*}}: \alpha(\nu) \backslash \pi_{t_{*}, x_{*}}^{(W)}\langle\nu \mid M, N\rangle \neq \emptyset
$$

We choose $\nu_{*} \in \mathcal{E}_{t_{*}}$ such that $\alpha\left(\nu_{*}\right) \backslash \pi_{t_{*}, x_{*}}^{(W)}\left\langle\nu_{*} \mid M, N\right\rangle \neq \emptyset$. Let

$$
\eta_{*} \in \alpha\left(\nu_{*}\right) \backslash \pi_{t_{*}, x_{*}}^{(W)}\left\langle\nu_{*} \mid M, N\right\rangle \text {. }
$$

By $(9.3), \eta_{*} \in \widetilde{\Pi}_{t_{*}}(\alpha)$ and, as a corollary (see $(9.15)$ ),

$$
\eta_{*} \in \mathcal{S}_{M, N}\left(t_{*}, x_{*}\right) .
$$

Using (9.9) and (9.17), we obtain $\eta_{*} \in \mathcal{H}_{t_{*}}$ and

$$
\Theta \triangleq\left\{\theta \in\left[t_{*}, \vartheta_{o}\right] \mid\left(\left(\theta, \varphi\left(\theta, t_{*}, x_{*}, \eta_{*}\right)\right) \in M\right) \&\left(\left(t, \varphi\left(t, t_{*}, x_{*}, \eta_{*}\right)\right) \in N \quad \forall t \in\left[t_{*}, \theta[)\right\} \neq \emptyset .\right.\right.
$$

Then, $\vartheta_{*} \triangleq \inf (\Theta) \in\left[t_{*}, \vartheta_{o}\right]$. Since $M \in \mathcal{F}$ and $\varphi\left(\cdot, t_{*}, x_{*}, \eta_{*}\right) \in C_{n}\left(\left[t_{*}, \vartheta_{o}\right]\right)$, the inclusion

$$
\left(\vartheta_{*}, \varphi\left(\vartheta_{*}, t_{*}, x_{*}, \eta_{*}\right)\right) \in M
$$

holds. From (9.18) and (9.19), we obtain $\vartheta_{*} \in \Theta$ (we use the property $\Theta \neq \emptyset$ ). In particular,

$$
\left(t, \varphi\left(t, t_{*}, x_{*}, \eta_{*}\right)\right) \in N \quad \forall t \in\left[t_{*}, \vartheta_{*}[.\right.
$$

Recall that $\eta_{*} \notin \pi_{t_{*}, x_{*}}^{(W)}\left\langle\nu_{*} \mid M, N\right\rangle$. Then, by (9.1), (9.10), and (9.16), $\forall \vartheta \in\left[t_{*}, \vartheta_{o}\right]$

$$
\left(\left(\vartheta, \varphi\left(\vartheta, t_{*}, x_{*}, \eta_{*}\right)\right) \notin M\right) \vee\left(\exists t \in \left[t_{*}, \vartheta\left[:\left(t, \varphi\left(t, t_{*}, x_{*}, \eta_{*}\right)\right) \notin W(M, N)\right) .\right.\right.
$$

In particular, from (9.19) and (9.21), we find that, for some $t^{*} \in\left[t_{*}, \vartheta_{*}[\right.$,

$$
\left(t^{*}, x^{*}\right) \notin W(M, N),
$$

where $x^{*} \triangleq \varphi\left(t^{*}, t_{*}, x_{*}, \eta_{*}\right)$. Certainly, by $(9.20),\left(t^{*}, x^{*}\right) \in N$. Thus, (9.22) implies the inclusion

$$
\left(t^{*}, x^{*}\right) \in N \backslash W(M, N) .
$$


By (9.23) and Theorem 3,

$$
\widetilde{\Pi}_{t^{*}}(\tilde{\alpha}) \backslash \mathcal{S}_{M, N}\left(t^{*}, x^{*}\right) \neq \emptyset \quad \forall \tilde{\alpha} \in \widetilde{A}_{t^{*}} .
$$

Thus, $t_{*} \in T, \alpha \in \widetilde{A}_{t_{*}}, \nu_{*} \in \mathcal{E}_{t_{*}}, \eta_{*} \in \Pi_{t_{*}}\left(\nu_{*}\right)$, and $t^{*} \in\left[t_{*}, \vartheta_{o}\right]$ (we use (9.16) and the obvious inclusion $\alpha\left(\nu_{*}\right) \subset \Pi_{t_{*}}\left(\nu_{*}\right)$; see (9.1)). Then, by (9.5),

$$
\bar{\alpha} \triangleq\left(\left\{\tilde{\eta} \in \Pi_{t^{*}}(\tilde{\nu}) \mid \eta_{*} \perp \tilde{\eta} \in \alpha\left(\nu_{*} \bowtie \tilde{\nu}\right)\right\}\right)_{\tilde{\nu} \in \mathcal{E}_{t^{*}}} \in \widetilde{A}_{t^{*}}
$$

Thus, $\bar{\alpha}: \mathcal{E}_{t^{*}} \longrightarrow \mathcal{P}^{\prime}\left(\mathcal{H}_{t^{*}}\right)$ and, in view of $(9.25)$, we obtain

$$
\bar{\alpha}(\nu)=\left\{\eta \in \Pi_{t^{*}}(\nu) \mid \eta_{*} \perp \eta \in \alpha\left(\nu_{*} \bowtie \nu\right)\right\} \quad \forall \nu \in \mathcal{E}_{t^{*}} .
$$

We recall that, by $(9.3), \widetilde{\Pi}_{t^{*}}(\bar{\alpha})$ is the union of all sets $\bar{\alpha}(\nu), \nu \in \mathcal{E}_{t^{*}}$. In addition, $\widetilde{\Pi}_{t^{*}}(\bar{\alpha}) \backslash$ $\mathcal{S}_{M, N}\left(t^{*}, x^{*}\right) \neq \emptyset$. Using (9.24) and (9.25), we choose

$$
\eta^{*} \in \widetilde{\Pi}_{t^{*}}(\bar{\alpha}) \backslash \mathcal{S}_{M, N}\left(t^{*}, x^{*}\right) .
$$

Then, $\eta^{*} \in \bar{\alpha}\left(\nu^{*}\right)$, where $\nu^{*} \in \mathcal{E}_{t^{*}}$. In particular, $\eta^{*} \in \mathcal{H}_{t^{*}}$. In addition, by $(3.9), \nu_{*} \bowtie \nu^{*} \in \mathcal{E}_{t_{*}}$, for which

$$
\left(\nu_{*} \bowtie \nu^{*}\right)(D)=\nu_{*}\left(D \cap \left(\left[t_{*}, t^{*}[\times Q)+\nu^{*}\left(D \cap Z_{t^{*}}\right) \quad \forall D \in \mathcal{D}_{t_{*}} .\right.\right.\right.
$$

From (9.26) and (9.27), we obtain

$$
\eta_{*} \perp \eta^{*} \in \alpha\left(\nu_{*} \bowtie \nu^{*}\right) .
$$

By (9.3), $\eta_{*} \perp \eta^{*} \in \widetilde{\Pi}_{t_{*}}(\alpha)$. From (9.15) and (9.28), the inclusion

$$
\eta_{*} \perp \eta^{*} \in \mathcal{S}_{M, N}\left(t_{*}, x_{*}\right)
$$

follows. Then, by (9.9) and (9.29), we obtain, for some $\theta_{o} \in\left[t_{*}, \vartheta_{o}\right]$,

$$
\left(\left(\theta_{o}, \varphi\left(\theta_{o}, t_{*}, x_{*}, \eta_{*} \perp \eta^{*}\right)\right) \in M\right) \&\left(( t , \varphi ( t , t _ { * } , x _ { * } , \eta _ { * } \perp \eta ^ { * } ) ) \in N \quad \forall t \in \left[t_{*}, \theta_{o}[) .\right.\right.
$$

In addition, $\left(t_{*}, x_{*}\right) \in T \times \mathbb{R}^{n}, \eta_{*} \in \mathcal{H}_{t_{*}}, t^{*} \in\left[t_{*}, \vartheta_{o}\right]$, and $\eta^{*} \in \mathcal{H}_{t^{*}}$. Moreover, $x^{*}=\varphi\left(t^{*}, t_{*}, x_{*}, \eta_{*}\right)$. Therefore, from the property (2) of Section 3 ,

$$
\begin{array}{r}
\left(\varphi\left(t, t_{*}, x_{*}, \eta_{*} \perp \eta^{*}\right)=\varphi\left(t, t_{*}, x_{*}, \eta_{*}\right) \quad \forall t \in\left[t_{*}, t^{*}\right]\right) \& \\
\&\left(\varphi\left(t, t_{*}, x_{*}, \eta_{*} \perp \eta^{*}\right)=\varphi\left(t, t^{*}, x^{*}, \eta^{*}\right) \quad \forall t \in\left[t^{*}, \vartheta_{o}\right]\right) .
\end{array}
$$

Recall that $t^{*}<\vartheta_{*}$. Since $\Theta \subset\left[\vartheta_{*}, \vartheta_{o}\right]$, we obtain $t \notin \Theta \forall t \in\left[t_{*}, t^{*}\right]$. By (9.20),

$$
\left(t, \varphi\left(t, t_{*}, x_{*}, \eta_{*}\right)\right) \in N \quad \forall t \in\left[t_{*}, t^{*}\right] .
$$

From (9.18) and (9.32), we obtain

$$
\left(t, \varphi\left(t, t_{*}, x_{*}, \eta_{*}\right)\right) \notin M \quad \forall t \in\left[t_{*}, t^{*}\right]
$$

(we use the property $\Theta \cap\left[t_{*}, t^{*}\right]=\emptyset$ ). By (9.31) and (9.33),

$$
\left(t, \varphi\left(t, t_{*}, x_{*}, \eta_{*} \perp \eta^{*}\right)\right) \notin M \quad \forall t \in\left[t_{*}, t^{*}\right] .
$$

Therefore (see (9.30) and (9.34)), $t^{*}<\theta_{o}$. Then, $\left[t^{*}, \theta_{o}[\neq \emptyset\right.$. By (9.31),

$$
\varphi\left(t, t_{*}, x_{*}, \eta_{*} \perp \eta^{*}\right)=\varphi\left(t, t^{*}, x^{*}, \eta^{*}\right) \quad \forall t \in\left[t^{*}, \theta_{o}[.\right.
$$

Recall that $\eta^{*} \in \mathcal{H}_{t^{*}} \backslash \mathcal{S}_{M, N}\left(t^{*}, x^{*}\right)$. Then, by (9.9) $\forall \theta \in\left[t^{*}, \vartheta_{o}\right]$,

$$
\left(\left(\theta, \varphi\left(\theta, t^{*}, x^{*}, \eta^{*}\right)\right) \notin M\right) \vee\left(\exists t \in \left[t^{*}, \theta\left[:\left(t, \varphi\left(t, t^{*}, x^{*}, \eta^{*}\right)\right) \notin N\right) .\right.\right.
$$


In particular, we obtain

$$
\left(\left(\theta_{o}, \varphi\left(\theta_{o}, t^{*}, x^{*}, \eta^{*}\right)\right) \notin M\right) \vee\left(\exists t \in \left[t^{*}, \theta_{o}\left[:\left(t, \varphi\left(t, t^{*}, x^{*}, \eta^{*}\right)\right) \notin N\right) .\right.\right.
$$

In addition, $\left.\left.\theta_{o} \in\right] t^{*}, \vartheta_{o}\right]$ and, by (9.31), $\varphi\left(\theta_{o}, t_{*}, x_{*}, \eta_{*} \perp \eta^{*}\right)=\varphi\left(\theta_{o}, t^{*}, x^{*}, \eta^{*}\right)$. Then, by (9.30),

$$
\left(\theta_{o}, \varphi\left(\theta_{o}, t^{*}, x^{*}, \eta^{*}\right)\right) \in M
$$

Therefore, from (9.36), we obtain, for some $t^{o} \in\left[t^{*}, \theta_{o}[\right.$,

$$
\left(t^{o}, \varphi\left(t^{o}, t^{*}, x^{*}, \eta^{*}\right)\right) \notin N
$$

As a corollary, the following property

$$
\left(t^{o}, \varphi\left(t^{o}, t_{*}, x_{*}, \eta_{*} \perp \eta^{*}\right)\right) \notin N
$$

holds. Since $t^{o} \in\left[t_{*}, \theta_{o}\left[\right.\right.$ (we recall that $t^{*} \in\left[t_{*}, \vartheta_{o}\right]$ ), by $(9.30)$,

$$
\left(t^{o}, \varphi\left(t^{o}, t_{*}, x_{*}, \eta_{*} \perp \eta^{*}\right)\right) \in N \text {. }
$$

From (9.38) and (9.39), we have the obvious contradiction. This contradiction proves that $\alpha \stackrel{t_{*}}{\sqsubseteq} \pi_{t_{*}, x_{*}}^{(W)}\langle\cdot \mid M, N\rangle$.

Thus, for $\left(t_{*}, x_{*}\right) \in W(M, N)$, the quasi-program $\pi_{t_{*}, x_{*}}^{(W)}\langle\cdot \mid M, N\rangle$ is the greatest element of the nonempty set

$$
\left\{\alpha \in \widetilde{A}_{t_{*}} \mid \widetilde{\Pi}_{t_{*}}(\alpha) \subset \mathcal{S}_{M, N}\left(t_{*}, x_{*}\right)\right\}
$$

in partially ordered space $\left(\widetilde{A}_{t_{*}}, \stackrel{t_{*}}{\sqsubseteq}\right)$.

We note that, in [18], a variant of realization of quasistrategies in the class of procedures with dynamic model was stated.

\section{Conclusion}

We proposed here a new variant of the program iterations method for solving differential guidance-evasion games.

The structure of set-valued quasistrategies solving the guidance problem is found and investigated for sufficiently general class of systems.

\section{REFERENCES}

1. Isaacs R. Differential Games. John Wiley, New York, 1965 P. xxvii +384 .

2. Krasovskii N.N. and Subbotin A.I. Game-Theoretical Control Problems. Springer-Verlag. New York, Berlin, Heidelberg, 1988.

3. Krasovskii N.N. and Subbotin A.I. An Alternative for the Game Problem of Convergence // J. Appl. Math. Mech. 1971. Vol. 34, no. 6. P. 948-965.

4. Chentsov A.G. On the structure of a game problem of convergence // Soviet Math. Dokl. 1975. Vol. 16. P. 1404-1406. [Russian]

5. Chentsov A.G. On a game problem of guidance // Soviet Mathematics, 1976. Vol. 17, no. 1. P. 73-77. [Russian]

6. Chentsov A.G. On a game problem of converging at a given instant of time // Mathematics of the USSR-Sbornik, 1976. Vol. 28 (3). P. 353-376. [Russian]

7. Chistiakov S.V. On solving pursuit game problems // J. of Applied Mathematics and Mechanics, 1977. Vol. 41, Issue 5. P. 855-852. doi:10.1016/0021-8928(77)90167-8

8. Ukhobotov V.I. Construction of a stable bridge for a class of linear games // J. of Applied Mathematics and Mechanics, 1977. Vol. 41, Issue 2. P. 350-354. doi:10.1016/0021-8928(77)90021-1 
9. Chentsov A.G. The programmed iterations method for differential game of guidance-deviation // Dep. in VINITI, № 1933-79, Sverdlovsk. [Russian]

10. Kryazhimskii A.V. On the theory of positional differential games of approach and evasion // Doklady AN SSSR, 1978. Vol. 239, no. 4. P. 779-782. [Russian]

11. Chentsov A.G., Morina S.I. Extensions and relaxations. Dordrecht; Boston; London: Kluwer Acad. Publ., 2002. 408 p.

12. Subbotin A. I. and Chentsov A. G. Optimizatsiya garantii v zadachakh upravleniya (Optimization of a Guarantee in Control Problems), Moscow: Nauka, 1981. 288 p. [Russian]

13. Chentsov A.G. About alternative in class of quasistrategies for differential game of guidance-deviation // Differential equations, 1980. Vol. XVI, no. 10. P. 1801-1808. [Russian]

14. Warga J. Optimal control of differential and functional equations. New York: Academic Press, 1972. P. xiv +531 .

15. Chentsov A.G. Elements of finitely additive theory of a measure, I// Ekaterinburg, UGTU-UPI, 2008. 389 p. [Russian]

16. Engelking R. General topology, PWN. Warsaw, 1977. 626 p.

17. Chentsov A. G. The programmed iterations method in game problem of guidance // Trudy IMM UrO RAN, 2016. Vol. 22, no. 2, P. 307-324.

18. Kryazhimskii A. V., Chentsov A. G. About structure of game control in problems of quidance and deviation // Dep. in VINITI № 1729-80, Sverdlovsk. [Russian] 